\title{
Estimation of Structural Properties of Online Social Networks at the Extreme
}

\author{
Emrah Cem, Kamil Sarac \\ Department of Computer Science, The University of Texas at Dallas, Richardson, Texas, \\ 75080-3021
}

\begin{abstract}
Sampling is a commonly used technique for studying structural properties of online social networks (OSNs). Due to privacy, business, and performance concerns, OSN service providers impose limitations on data access for third parties. The implication of this practice is that one needs to come up with an applicable sampling scheme that can function under these limitations to efficiently estimate structural properties of interest. In this paper, we study how accurately some important properties of graphs can be estimated under a limited data access model. More specifically, we consider random neighbor access (RNA) model as a rather limited data access model in OSNs. In the RNA model, the only query available to get data from the studied graph is the random neighbor query which returns the id of a random neighbor for a given vertex id. We propose various sampling schemes and estimators for average degree and network size under the RNA model. We conduct extensive experiments on both real world OSN graphs and synthetic graphs (1) to measure the performance of the proposed estimators and (2) to identify the factors affecting the accuracy of our estimators. We find that while the average degree estimators can make accurate estimations with reasonable sample sizes despite the extreme data access limitations of the RNA model, network size estimators require quite large sample sizes for accurate estimations.
\end{abstract}

Keywords: estimation, sampling, online social networks

Email addresses: emrah.cem@utdallas.edu (Emrah Cem), ksarac@utdallas.edu (Kamil Sarac)

Preprint submitted to Computer Networks

July 20, 2016

(C) 2015. This manuscript version is made available under the Elsevier user license http://www.elsevier.com/open-access/userlicense/1.0/ 


\section{Introduction}

Online social networks (OSNs) such as Facebook, Twitter, and LinkedIn have become an important part of our daily life. They not only create a platform to connect people but also provide a means to analyze and characterize the behavior of their users. Estimating the structural properties of the underlying networks of OSNs using the statistical estimation theory has drawn a lot of attention [1-9]. Understanding the structure of these OSN graphs might help to develop algorithms for detecting trusted or influential users [10, 11]; mitigate email spam [12]; defend against Sybil attacks [13]; and improve Internet search [14].

The main challenge in estimating the characteristics of OSNs for third parties is the limited access to the data of interest through a public API. In principle, sampling enables us to make population level estimations if data is collected with known statistical properties. However, limited data access can make it challenging to develop practical sampling schemes with known statistical properties. Data access limitation in OSNs is generally in two different forms: 1) query rate limitation and 2) authorization. Query rate limitation means that the number of API calls that can be made within a specified time period is limited by the OSN service provider. This limitation affects how fast the graph can be sampled. The practitioners generally partially overcome this limitation by using a parallel crawling approach to collect the data $[8,15]$. Even if the entire dataset is accessible and parallel crawling is used, accessing all the data is generally not efficient and sampling is a common way of increasing the efficiency at the expense of accuracy. Authorization is also an important feature of today's OSN services as it provides privacy for the OSN users. However, this feature makes statistical estimation impossible without making further assumptions about the unobserved data.

In this paper, we consider the estimation problem with a limited data access model called the random neighbor access (RNA) model. The only public API query that is available under the RNA model is the random neighbor query (RN-QUERY). The RN-QUERY returns the id of a neighbor selected uniformly at random and independently for a given vertex id (see Fig. 1). The RNA model is relevant because OSN service providers are imposing more and more limitations for third party access to their data and users are becoming more aware of privacy issues and share the data with limited number of people [16]. As one of the possible extreme cases of this trend, we consider the RNA model. Our main motivation is to investigate 

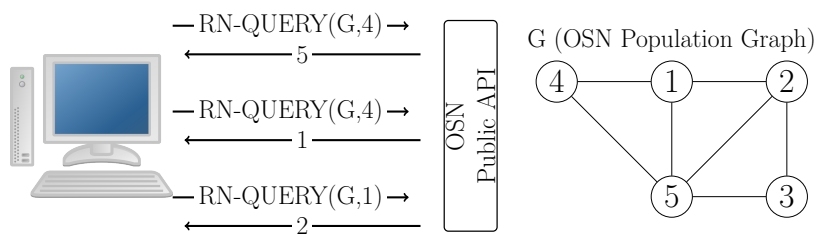

Figure 1: An example data collection from the OSN graph using the RN-QUERY calls. Note that we call the RN-QUERY twice for the vertex with id 4 and get different neighbors by chance.

the predictability of important structural properties under the RNA model. Although the model allows very limited data access, the probabilistic nature of the access model to the data allows us to make statistical estimations without further assumptions on the data. Studying the RNA model helps us understand the performance limitations of the estimators developed for an extreme scenario. In this paper, we specifically focus on the estimation of two important characteristics namely the average degree and the network size.

Sampling has been commonly used in OSN contexts but existing schemes are not applicable under the RNA model. Random walk (RW) [7, 8, 17-23] is the most commonly used sampling scheme in OSNs. It is well-known that the simple random walk (SRW) sampling in which a neighbor is selected uniformly at random at each step is biased towards high degree nodes, but its bias is known and can be corrected for connected and non-bipartite graphs using the vertex degrees $[24,25]$. An implicit assumption in the previous works is that the degrees of vertices visited by SRW are observable. However, we only observe the ids of the visited vertices under the RNA model. Therefore, previous estimators are not directly applicable. More recent studies $[9,19,20,22]$ propose various versions of RWs to improve the efficiency of the estimator for certain graphs. However, these estimators are also not directly applicable under the RNA model as the efficiency is generally achieved by assuming access to more data such as the availability of independent node sampling. Breadth-first search (BFS) and depth-first search (DFS) sampling methods are also easy to apply on OSNs, but it is not known how to correct their bias towards high degree nodes for arbitrary graphs [26, 27]. They are also not very efficient under the RNA model as the neighbor selection is not deterministic under the RNA model.

The main contributions of this paper are as follows: 


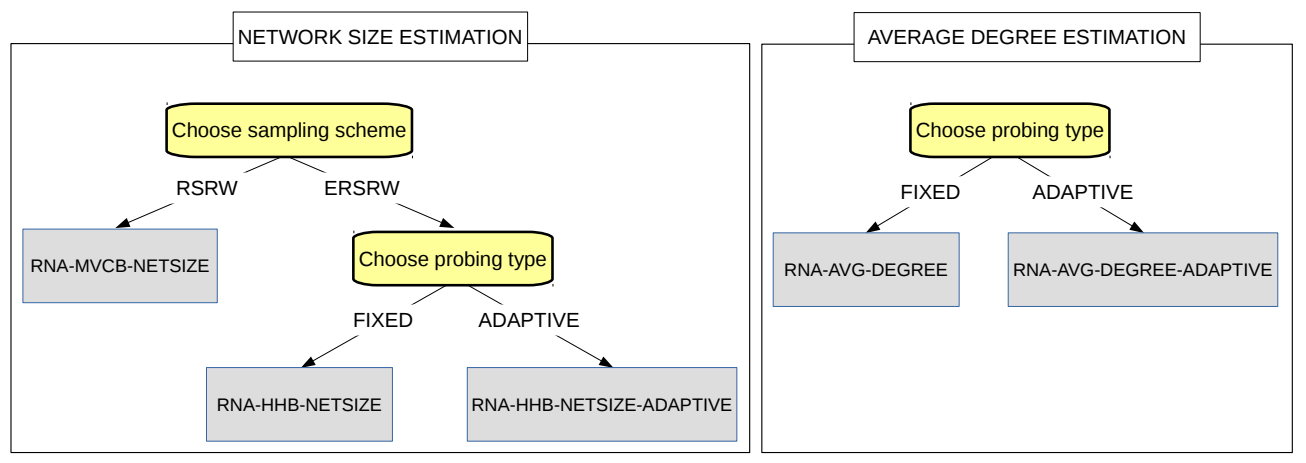

Figure 2: Proposed Estimators: Illustration of which estimator is used based on the sampling scheme and probing type. Probing type is applicable only on ERSRW sampling scheme. We propose average degree estimator under only ERSRW sampling, so choosing the sampling scheme step is not applicable.

1. We propose various sampling schemes and corresponding average degree and network size estimators under a rather limited probabilistic data access model called RNA model.

2. We propose an estimator for the reciprocals of the vertex degrees which we use in the estimation of the average degree and the network size properties. We provide both theoretical analysis and simulation based experiments on its accuracy.

3. We conduct extensive simulation studies on both real world and synthetic graphs (1) to measure the performance of the proposed estimators and (2) to study the factors that affect the accuracy of these estimators.

Our main findings are as follows:

1. The network size estimation is a challenging task under the RNA model and requires large number of RN-QUERY calls (e.g., more than the actual network size, even 40-50 times the network size in some cases). However, the average degree estimation can be done efficiently with the selection of a reasonable sampling design parameter.

2. Applying dependency reduction technique to the samples does not necessarily improve the estimation performance ${ }^{1}$. For instance, in the estimation of average degree, dependency reduction technique reduces the

\footnotetext{
${ }^{1}$ We use the term estimation performance as a combined measure of precision and
} 
precision of the estimation; while in the estimation of the network size, it increases the accuracy, especially when the sampling fraction $f>1$.

3. The dynamic nature of the underlying graph adds one more layer to the complexity of the estimation problem. The accuracy of the estimation is limited by how fast the samples can be collected and how fast the property of interest changes. As opposed to the static graph case, larger sample sizes do not provide better estimation results especially when the property of interest increases or decreases over time as the old data becomes unrepresentative of the current data.

The outline of the paper is as follows: Section 2 presents the background and the related work. Section 3 presents the RNA model and sampling designs. Section 4 presents the estimators for the RNA model. Section 5 presents our experimental evaluations. Section 6 discusses the practical issues. Section 7 concludes the paper.

\section{Background and Related Work}

The RNA model enables us to perform a walk, but not a SRW ${ }^{2}$, on the underlying graph. Nevertheless, the estimation techniques proposed under SRW sampling form the basis for those under the RNA model as the sampling schemes that we use under the RNA model, namely RSRW and ERSRW, are the modifications of the SRW. In this section, we first introduce the graph and sampling terminologies. Then, we briefly discuss SRW sampling and present the well-known and the state-of-the-art estimators for the average degree and network size properties under SRW sampling. We also discuss graph traversal methods (e.g., breadth-first search (BFS) and depth-first search (DFS)) and two relevant Monte Carlo (MC) methods and explain why they are not practical or applicable under the RNA model.

\subsection{Graph Terminology}

We denote an OSN graph by $G=(V, E)$ where $V$ is the finite set of nodes/vertices and $E$ is the set of links/edges. We use $N=|V|$ and $M=|E|$

accuracy. Precision is a measure of the stability (hence inversely proportional to the variance of the estimator) while accuracy is a measure of being close to the true parameter (hence proportional to the bias of the estimator) on the average.

${ }^{2}$ In SRW, the vertex degrees of visited vertices are assumed to be known. 
to denote the network size and the number of edges, respectively. We use $d_{v}$ to denote the degree of $v \in V$. We use $\tau_{d}=\sum_{u \in V} d_{u}$ and $\mu_{d}=\frac{\tau_{d}}{N}$ to denote the total and the average degree of $G$, respectively.

\subsection{Sampling Terminology}

We use $S$ and $s_{i}$ to denote the list of sampled nodes and the $i^{\text {th }}$ sampled node, respectively. We use $n=|S|$ and $f=n / N$ to denote the sample size and the sample fraction, respectively. We use $\mathscr{S}$ to denote the set of distinct vertices in $S$. We use $\Delta_{u}$ to denote the number of times vertex $u$ is sampled and call it the multiplicity of vertex $u$. We say that a collision occurs when the $i^{\text {th }}$ and the $j^{\text {th }}$ sampled vertices are the same for $i<j$.

Finally, we use $C=\sum_{u \in \mathscr{S}}\left(\begin{array}{c}\Delta_{u} \\ 2\end{array}\right)$ to denote the total number of collisions in the sample. We use $\left(\begin{array}{l}n \\ k\end{array}\right)$ to denote the binomial coefficient, i.e., the number of ways of picking $k$ unordered outcomes from $n$ possibilities. For instance, if $S=\{u, v, t, u, t, u\}$, then $C=\left(\begin{array}{l}3 \\ 2\end{array}\right)+\left(\begin{array}{l}1 \\ 2\end{array}\right)+\left(\begin{array}{l}2 \\ 2\end{array}\right)=4$ since $\Delta_{u}=3, \Delta_{v}=1$, and $\Delta_{t}=2$.

\subsection{SRW Sampling Design}

RW is a finite state time-reversible Markov chain and it has a wellestablished theory [28]. RW starts from a vertex and repeatedly moves to a random neighbor node. If the neighbor node is selected uniformly at random, it is called an SRW $[22,29]$. More formally, let $\left\{s_{t}\right\}$ be the Markov chain representing the sequence of sampled nodes by SRW. The transition probability matrix $\boldsymbol{P}_{\boldsymbol{S R W}}=\left\{p_{u v}\right\}_{u, v \in V}$ of SRW, where $p_{u v}$ is the probability of moving from vertex $u$ to vertex $v$, is given by

$$
p_{u v}=\left\{\begin{array}{ll}
1 / d_{u} & \text { if }(u, v) \in E \\
0 & \text { otherwise }
\end{array} .\right.
$$

Researchers develop variations of RW such as Metropolis-Hastings RW [30, 31], multiple dependent RW [19], multigraph RW [9], RW with jumps [32, 33] and weighted RW [7] to sample certain types of graphs more efficiently. However, the RNA model does not allow to make these modifications efficiently. For instance, they require independent node selection and/or the knowledge of the degrees of both the current node and the candidate node in order to efficiently choose the next node in the RW (see Eq. 8).

Stationary distribution of a walk is an important concept that defines the probability of being at any particular vertex. The fundamental theorem 
of Markov chains[34] states that every finite, irreducible, aperiodic Markov chain has a unique stationary distribution. Irreducibility and aperiodicity are achieved on connected and non-bipartite graphs. A walk is called stationary random walk if the initial vertex in the walk is selected from the stationary distribution $\pi=\left\{\pi_{u}\right\}_{u \in V}=\left\{d_{u} / 2 M\right\}_{u \in V}$. In this paper, we only consider stationary random walks. Even though the initial vertex selection from the stationary distribution is not trivial, we briefly discuss how it is generally achieved by practitioners in Section 6 .

Another important property of a RW is that there is a strong dependence on the consecutive samples. Thus, using estimators that assume independent vertex selection may yield inaccurate results if the samples obtained by a random walk are used directly. Kurant et al. [6] discuss some practical dependency reduction techniques such as safety-margin and shifted-thinning. In the safety-margin technique with parameter margin, the information obtained from the pairs of sampled nodes $s_{k}$ and $s_{l}$ that are close to each other, i.e., $|k-l| \leq$ margin, is ignored and the estimator is adjusted accordingly. For instance, the information might be whether there is a collision or not. In the shifted-thinning technique with thinning parameter $t$, sampled vertices are divided into $t$ groups. The first group contains every $t^{t h}$ sample starting from the first sample, the second group contains every $t^{\text {th }}$ sample starting from the second sample, and so on. Then, an estimation is performed for each group independently and the average of $t$ estimations is considered as the final estimation. We use these techniques in our estimators to reduce the dependency in the sample.

\subsection{Estimators for SRW Sampling Design}

In this section, we introduce the existing estimators for the average degree and network size properties when the data is collected by a SRW sampling and the degree information is observable at the sampled vertices. The estimators are not exhaustive but gives an overview of the known statistical techniques and recent advancements. The estimators that we introduce here form the basis for our estimators. We adapt the ideas presented in this section to the RNA model in Section 4.

\subsubsection{Average Degree Estimation}

The GENERALIZED estimator [35], a.k.a. self normalized importance sampling, is one of the most popular estimators for the average characteristics such as average age of users or average number of friends. Even though 
it is not restricted for vertex characteristics, we only discuss it for vertex characteristics for simplicity. It requires that the selection probability of each sampled vertex is independent, strictly positive and is known up to a constant $^{3}$. It is an asymptotically unbiased estimator and is defined as

$$
\hat{\mu}_{y}=\frac{\sum_{u \in S} y_{u} / w_{u}}{\sum_{u \in S} 1 / w_{u}},
$$

where $S$ and $y_{u}$ are the list of sampled nodes and the property of interest, respectively. $w_{u}$ is the selection weight of vertex $u$, i.e., the selection probability of vertex $u$ up to a constant.

In a stationary SRW, vertex $u \in V$ is sampled with probability $d_{u} / 2 M$. Therefore, we can select $w_{u}$ as $d_{u}$. As we estimate the average degree, we replace $y_{u}$ with $d_{u}$ and get the following estimator:

$$
\hat{\mu}_{d}=\frac{n}{\sum_{u \in S} 1 / d_{u}} .
$$

The issue with this estimator is the dependency of the sampled vertices. The ergodic theorem[34] states that the effect of the dependence in the sample disappears in the limit of the large sample size. However, we reduce the dependency of the sampled vertices by applying a shifted-thinning [6] to the random walk as we are interested in the finite sample sizes. Applying shiftedthinning to Eq. 3 gives the following average degree estimator which we call SRW-AVG-DEGREE:

$$
\hat{\mu}_{d}^{s t}=\frac{1}{t} \sum_{i=1}^{t}\left(\sum_{j=1}^{n / t} \frac{n / t}{1 / d_{s_{i+(j-1) t}}}\right),
$$

where $s_{i}$ denotes the $i^{t h}$ sampled node. Note that Eq. 4 with $t=1$ is equivalent to Eq. 3.

Note that SRW-AVG-DEGREE estimator still requires the degree information at the sampled vertices; hence, it is not directly applicable under the RNA model. In Section 4.2, we present an estimator called RNA-AVGDEGREE estimator that modifies SRW-AVG-DEGREE estimator to estimate the average degree by using only RN-QUERY calls.

\footnotetext{
${ }^{3}$ An alternative is the Hansen-Hurwitz (HH) estimator which additionally requires the network size and the exact value of the selection probability of each sampled vertex.
} 
A recent study [1] proposes an average degree estimator under a prescribed distribution and shows how to realize it using the samples collected by a modified RW. However, realizing the prescribed distribution under the RNA model is not efficient as the accuracy highly depends on the knowledge of the vertex degrees. Therefore, we do not consider to adapt the estimator into the RNA model.

Another recent study by Lu et al. [36] shows that random edge (RE) sampling and its corresponding harmonic mean estimator, which we refer as GENERALIZED estimator in this paper, can reduce the estimation variance significantly as compared to random vertex sampling. The study also shows that although RW sampling is an approximation to the random edge sampling, harmonic mean estimator under random edge sampling always outperforms the same estimator under RW sampling. Although these are important theoretical findings, independent sampling schemes such as random edge sampling and random vertex sampling are not applicable under the RNA model and we focus on how well we can make estimations under the constraints of the RNA model although it depends on the graph conductance.

\subsubsection{Network Size Estimation}

In this section, we present commonly used and the state of the art network size estimators. We discuss why they are not directly applicable under the RNA model.

Hansen-Hurwitz (HH) estimator is a design-unbiased estimator for any total that can be represented as a sum of local property values under unequalprobability, independent, and with-replacement sampling designs. More formally, let $\tau_{y}=\sum_{u \in V} y_{u}$, then $\mathrm{HH}$ estimator for $\tau_{y}$ is given by

$$
\hat{\tau}_{y}=\frac{1}{n} \sum_{u \in S} \frac{y_{u}}{\pi_{u}} .
$$

We can obtain a network size estimator by setting $y_{u}=1$ and $\pi_{u}=\frac{d_{u}}{2 M}$ for all $u \in S$ :

$$
\hat{N}=\frac{2 M}{n} \sum_{u \in S} \frac{1}{d_{u}}=\frac{2 M}{\hat{\mu}_{d}} .
$$

Note that this is a natural estimator as $N=2 M / \mu_{d}$. One of the issues with this estimator is that $M$ is usually not known. In Section 4.3.2, we propose an estimator for $M, \hat{M}$, under the RNA model (see Eq. 11). Therefore, we can get a network size estimator, which we call SRW-HHB-NETSIZE estimator, 


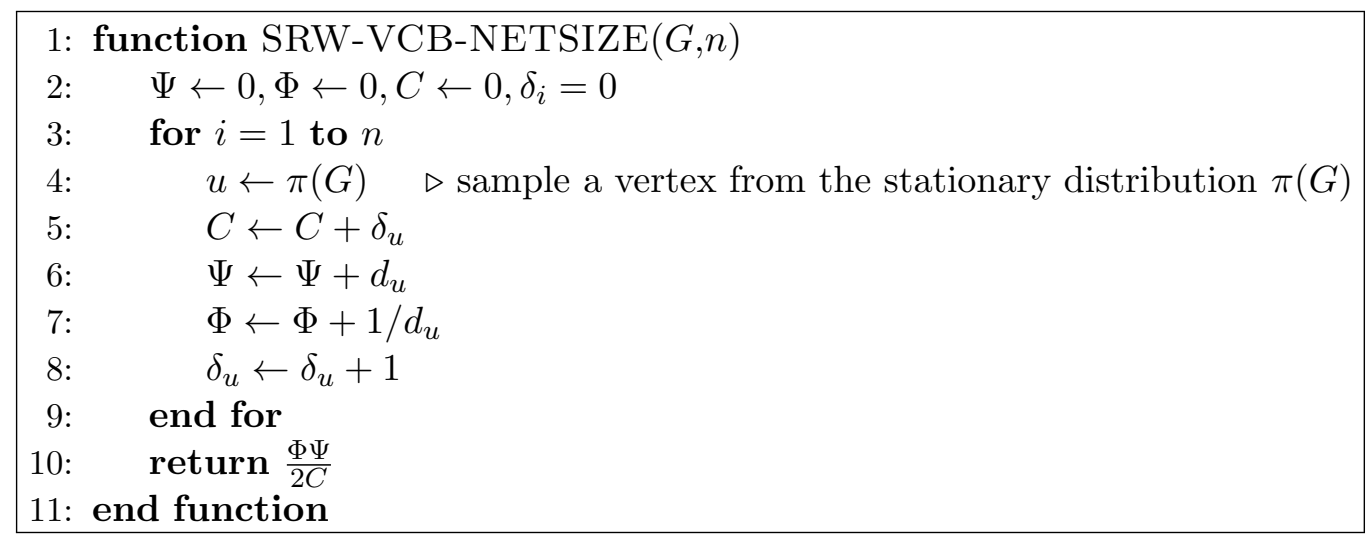

Algorithm 1: Vertex collision based network size estimator under SRW sampling (SRWVCB-NETSIZE) by Katzir et al. [2]

by replacing $M$ with $\hat{M}$ in Eq. 6 and $\hat{\mu}_{d}$ with $\hat{\mu}_{d}^{s t}$. Thus, SRW-HHB-NETSIZE estimator becomes

$$
\hat{N}=\frac{2 \hat{M}}{\hat{\mu}_{d}^{s t}} .
$$

Given that $\hat{\mu}_{d}^{s t}$ requires the vertex degrees (see Eq. 4), SRW-HHB-NETSIZE estimator is not directly applicable under the RNA model. In Section 4.3.2, we propose an estimator called RNA-HHB-NETSIZE estimator that modifies SRW-HHB-NETSIZE estimator to estimate the network size by using only RN-QUERY calls.

Another estimator that is commonly used, especially in ecology, is called mark-and-recapture (also known as capture-recapture) estimator [37]. To use mark-and-recapture estimator, we first select $k_{1}$ samples from the population uniformly at random without replacement, and then mark and release them to the population. Then, we select $k_{2}$ samples from the same population uniformly at random without replacement. Let $c$ denote the number of marked units selected in the second sample. Then, mark-and-recapture estimator for network size, which is asymptotically unbiased, is given by $\hat{N}_{M R}=k_{1} k_{2} / c$. As the estimator requires uniform node selection which is very inefficient under the RNA model(see 2.6), this estimator is not practical under the RNA model.

In a recent study [2], Katzir et al. propose a vertex collision based estimator for network size, which we call SRW-VCB-NETSIZE, when the vertices are sampled independently from the stationary distribution of the 
graph $G$, denoted by $\pi(G)$ (see Algorithm 1). They show that the SRWVCB-NETSIZE estimator is approximately asymptotically unbiased for the network size. The SRW-VCB-NETSIZE estimator keeps track of the sum of degrees $\left(\Psi=\sum_{u \in S} d_{u}\right)$, the sum of inverse degrees $\left(\Phi=\sum_{u \in S} \frac{1}{d_{u}}\right)$, and the total number of collisions $C$ in the sample (between line 3 and 9 ) and uses these values to estimate the network size. Note that we use $\delta_{s_{i}}$ to keep the number of times $s_{i}$ is sampled before iteration $i$ and use this variable to compute the total number of collisions on the fly. They show that the performance of the proposed estimator under this sampling design is superior to the collision-based estimator under the sampling design that selects vertices independently and uniformly at random. Note that the SRW-VCB-NETSIZE estimator uses the samples collected independently from the stationary distribution of the underlying graph and their degrees. As both of these are not available under the RNA model, SRW-VCB-NETSIZE is not applicable under the RNA model. In Section 4.3.1 we propose RNA-MVCB-NETSIZE estimator, an adapted version of the SRW-VCB-NETSIZE estimator, that is applicable under the RNA model. RNA-MVCB-NETSIZE estimator uses vertex multiplicities of sampled vertices instead of vertex degrees and utilizes only the samples that are not highly correlated in the random walk.

In another study, Lu et al. [38] characterize the relative bias of a collision based network size estimator $\hat{N}_{c}=\left(\gamma^{2}+1\right)\left(\begin{array}{l}n \\ 2\end{array}\right) \frac{1}{C}$ under SRW sampling, where $\gamma$ is the coefficient of variation of the degrees. Using a similar approach, we can estimate the degree of a vertex using $\hat{d}_{v}=\left(\begin{array}{c}\omega \\ 2\end{array}\right) \frac{1}{c}$, where $\omega$ and $c$ are the number of RN-QUERY calls made for the vertex $v$ and the number of collisions among the neighbors of vertex $v$, respectively. However, in our estimators for both network size and average degree, we use RNA-REC-DEGREE estimator (see Algorithm 2) which directly estimates the reciprocals of the degrees and hence does not have the bias issue discussed by Lu et al. The bias in our estimators for average degree and network size are originated from the GENERALIZED and HH estimators discussed in Section 2.4.

In a more recent study, Hardiman et al. [3] propose a vertex neighbor collision based network size (SRW-VNCB-NETSIZE) estimator using the weighted neighbor collisions and the safety-margin technique so that sampled vertices will be approximately uncorrelated. More specifically, the estimator uses the number of common neighbors of approximately uncorrelated node pairs. They show that the estimation performance of SRW-VNCBNETSIZE is better than that of SRW-VCB-NETSIZE estimator. However, SRW-VNCB-NETSIZE estimator requires knowing not only the degrees of 
the sampled vertices but also the ids of the neighbors of the visited vertices. This is very inefficient under the RNA model. Therefore, we do not consider the adapted version of SRW-VNCB-NETSIZE estimator as a practical estimator under the RNA model.

\subsection{Graph Traversal Methods}

Graph traversal methods are alternative crawling-based graph sampling algorithms. Unlike RW, they sample the graph without replacement. BFS [3943], DFS [39, 44], Forest-Fire (FF) [33], and Snowball sampling [45] are popular graph traversal methods. All these graph traversal methods have biases that are hard to characterize for general graphs. For instance, the studies by Kurant et al. [26, 27] focus on the bias of BFS in random graphs with arbitrary degree distribution. The characterization of the bias in other graph traversal algorithms is still an active research area and is beyond the scope of this paper.

\subsection{Monte-Carlo Methods}

The basic idea in all Monte-Carlo (MC) methods is to sample the data from an easy-to-sample distribution (i.e., proposal distribution), and use these samples to simulate the target distribution. We briefly discuss two MC methods: Rejection Sampling (RS) [46] and Metropolis-Hastings RW (MHRW) [47]. In the context of this paper, the target distribution is the uniform distribution as we compute average and the sum ${ }^{4}$. In order to select one sample from the target distribution, several samples might be collected from the proposal distribution and this may make the MC method inefficient. We next discuss why RS and MHRW are inefficient under the RNA model.

The rejection sampling (a.k.a. accept-reject sampling) is a popular MC method to get samples from an arbitrary distribution and can be used to get a uniform sample of vertex ids by randomly generating a vertex id and checking if RN-QUERY succeeds (returns a neighbor id). If RN-QUERY succeeds, the vertex id is included in the sample; otherwise, it is discarded. Obviously, the rejection sampling is efficient only if the vertex id space is dense which is not the case in the context of this paper (see Section 3.1, Assumption 5).

MHRW is a Markov Chain Monte Carlo (MCMC) method that can remove the bias introduced by SRW by rearranging the one step transition

\footnotetext{
${ }^{4}$ Network size can be considered as the sum over nodes with local value one.
} 
probability matrix of SRW. Intuitively, for a large enough sample size, the walk visits each vertex in the graph with equal probability. More specifically, the transition probability matrix $\boldsymbol{P}_{\boldsymbol{M H} \boldsymbol{H} \boldsymbol{W}}=\left\{p_{u v}\right\}_{u, v \in V}$ of MHRW is given by

$$
p_{u v}= \begin{cases}\min \left(1, \frac{d_{u}}{d_{v}}\right) & \text { if }(u, v) \in E \\ 0 & \text { if } u \neq v,(u, v) \notin E\end{cases}
$$

and $p_{u u}=1-\sum_{u \neq v} p_{u v}$. MHRW can be implemented in two-steps to select the next sample. In the first step, a candidate vertex $v$ is selected uniformly at random among the neighbors. In the second step, $v$ is accepted as a sample with probability $p_{u v}$, otherwise $u$ is accepted as a sample. The transition probabilities of MHRW depend on the degrees of both the current vertex and the proposed vertex. In the RNA model, these are not available and estimating both is not very efficient especially when the candidate vertex is rejected in the second step since all RN-QUERY calls made to estimate the degree of the candidate vertex might be wasted.

\subsection{Other Related Work}

Estimation of the structural properties of graphs has been popular in social sciences, especially in the survey sampling field, during 1970s and 1980s [48-54]. These papers have introduced the statistical methodology for estimating properties of graphs based on various simple sampling methods such as induced subgraph sampling based on random vertex selection, random edge sampling, and star sampling [54]. There are also studies that propose link-tracing sampling methods and inference techniques for hardto-reach populations such as snowball-sampling [55] and respondent-driven sampling $[24,25,56-59]$. Additionally, there are studies focusing on estimation of the properties of ego-centric networks rather than the properties of the complete networks $[60,61]$.

Recently, there has been an interest in the development of various randomwalk based sampling methods in the sampling and estimation problems in large scale real world networks such as OSN, web, and search engines $[3,4$, $7,8,18-21,32,62-65]$.

The research community has also been interested in studying various characteristics of such large scale networks including degree distribution [19, 21, 33, 66-69], clustering coefficient [3, 19, 21, 68, 70], power-law exponent [39], average path length $[71,72]$, centrality measures $[68,71]$, and diameter $[33$, 39]. 
Researchers have also studied the task of how to choose an effective sampling scheme, how to evaluate different sampling schemes for studying a particular estimation problem [33, 70], and the impact of underlying graph structure and the studied graph property on the effectiveness of the sampling algorithms [73].

\section{The RNA Model and Sampling Designs}

We introduce the RNA model through the assumptions on the underlying graph and the data access limitations to the underlying graph. We also present the proposed sampling designs for the RNA model.

\subsection{The RNA Model}

The RNA model consists of the following assumptions about the underlying graph and the data access limitations:

1. The underlying graph is static.

2. The underlying graph is connected, non-bipartite, and undirected.

3. The graph is accessible only through the RN-QUERY calls. RN-QUERY returns one of the neighbors uniformly at random for a given valid vertex id $i$. We say that RN-QUERY fails if $i$ is not a valid vertex id.

4. We initially have access to one of the valid node ids.

5. The vertex id space is sparse, the number of valid ids is much less than the size of the id space.

The first assumption is for mathematical convenience. Providing bounds for estimators on dynamic graphs require modeling the dynamics of the network which is beyond the scope of this paper [31, 74, 75]. However, we still provide a simulation experiment and discuss the factors affecting the accuracy of the estimators under dynamic networks (see Section 6.3). The second assumption is required for the estimators to have good asymptotic properties. ${ }^{5}$ The third assumption is the restriction on the data collection from the underlying graph. The fourth assumption is to get the first user id in the walk. The last assumption does not affect our estimation as we perform a walk on the graph. However, it makes estimation through rejection sampling

\footnotetext{
${ }^{5}$ These are the requirements of the underlying graph for the random walk to have a stationary distribution.
} 
u $\left(\mathrm{v}: i^{\text {th }} \mathrm{RN}\right.$-QUERY call is made for vertex id $\mathrm{u}$ and it returns vertex id $\mathrm{v}$.

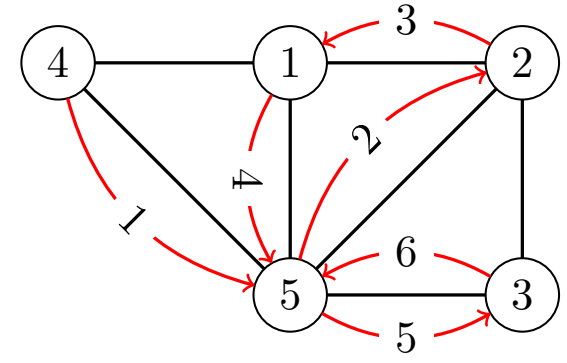

(a) Illustration of RSRW

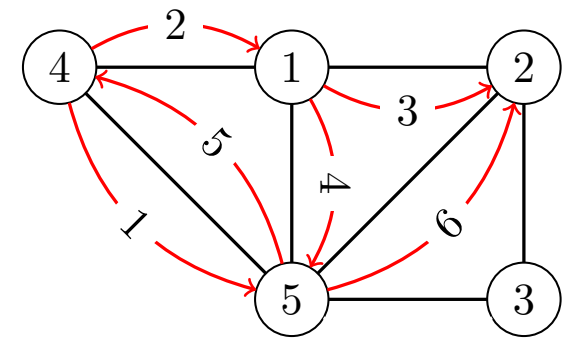

(b) Illustration of ERSRW with $\omega=2$

Figure 3: Illustration of the proposed sampling designs with a sample size of 6 on a small population graph.

impractical; otherwise one can simply generate a random id and make an RN-QUERY using that id to get an independent sample from the graph. In fact, the last assumption is very common with popular OSNs. For instance, Facebook has increased the id space of its users from 32 bit to 64 bit in 2009 and hence the id range has increased from $\left[0,2^{32}\right]$ to $\left[0,2^{64}\right]$. The implication of this is that most of the RN-QUERY calls fail if random ids are selected from the id range and RN-QUERY calls are made.

\subsection{Sampling Designs}

We introduce two sampling designs that are applicable under the RNA model. The adaptive version of the ERSRW sampling design is discussed in Section 6.2 together with the corresponding estimators.

\subsubsection{Restricted Simple Random Walk (RSRW)}

In a typical simple random walk (SRW) setting, it is assumed that the degrees of the visited vertices are observed. Under the RNA model, we can still perform a random walk on the underlying graph but we only observe the vertex ids of the visited vertices. We call this version of the random walk as the RSRW. Note that the restriction refers to the ability to observe only the vertex ids not to the ability to perform a random walk. Fig. 3a shows an example RSRW on a small population graph. The obtained sample is $S=\{5,2,1,5,3,5\}$. The challenge here is to propose an estimator using only the information in $S$. 


\subsubsection{Exploratory Restricted Simple Random Walk With Fixed Probing (ERSRW)}

Note that RSRW goes to a neighbor vertex after every RN-QUERY call. An alternative sampling scheme is to make several RN-QUERY calls for the current vertex before going to a neighbor vertex. The aim of making several RN-QUERY calls for the same vertex is to estimate the reciprocal of the vertex degree which is used to correct the sampling bias.

We make a constant number $\omega$ of RN-QUERY calls for the current vertex before going to a neighbor vertex in the walk. Fig. 3b shows an example ERSRW on a small population graph. The obtained sample is $S=$ $\{5,1,2,5,4,2\}$. Note that we make two RN-QUERY calls for vertices 4, 1 , and 5 and we only go to the neighbor vertex in the $\omega^{\text {th }}$ RN-QUERY call. For instance, the first RN-QUERY call returns id 1, but we do not go to that vertex and perform an RN-QUERY for that vertex since $\omega \neq 1$. In general, for a given $\omega$ and $n$, we basically perform an RSRW of size $\lfloor n / \omega\rfloor$ and we sample $\omega$ neighbors of each vertices with replacement in the walk ${ }^{6}$.

\section{Estimation under the RNA Model}

In this section we present our estimators that use the data collected by the sampling designs we introduced in Section 3.2. Table 1 shows how our estimators for the RNA model differs from the estimators for the SRW model. The estimators shown in the table do not use the dependency reduction techniques for the simplicity of comparison. We propose RNA-AVG-DEGREE estimator for the average degree. RNA-AVG-DEGREE estimator uses the data collected by ERSRW sampling. We propose two different estimators for the network size : 1) RNA-MVCB-NETSIZE and 2) RNA-HHB-NETSIZE. RNA-MVCB-NETSIZE estimator uses the data collected by RSRW sampling while RNA-HHB-NETSIZE estimator uses the data collected by ERSRW sampling.

Before proceeding with the average degree and network size estimators, we first introduce an estimator for the reciprocal of the vertex degrees that uses the data collected by ERSRW sampling. We use this estimator in both RNA-AVG-DEGREE and RNA-HHB-NETSIZE estimators to correct the

\footnotetext{
${ }^{6}$ If $n$ is not divisible by $\omega$, we ignore the last $(n \bmod \omega)$ RN-QUERY calls made for the last vertex in the random walk.
} 
sampling bias due to random walk. Next, we give the details of the average degree and network size estimators.

\subsection{On-the-fly Estimator for the Reciprocal of the Vertex Degree: RNA-REC-DEGREE}

Given that SRW-AVG-DEGREE and SRW-HHB-NETSIZE estimators require the reciprocal of the vertex degrees which is not available under the RNA model, we need to estimate them. In this section, we propose an estimator for the reciprocal of the vertex degree based on the sample collected by ERSRW sampling method. The main motivation is that the RNA model allows us to perform a random walk which visits vertices with higher degrees with higher probability. Therefore, the plug-in estimator gives an overestimation, but this bias can be corrected if the vertex degrees are estimated accurately. To estimate the vertex degree, we sample the neighbor ids of the queried vertex independently and uniformly at random with replacement by making several RN-QUERY calls for the same vertex. Then, we estimate the reciprocal of the vertex degree, based on the number of RN-QUERY calls and collisions. We make a constant number of RN-QUERY calls per sampled vertex and denote it by $\omega$ (see Algorithm 2). We discuss the version with adaptive number of RN-QUERY calls per sampled vertex in Section 6.2.

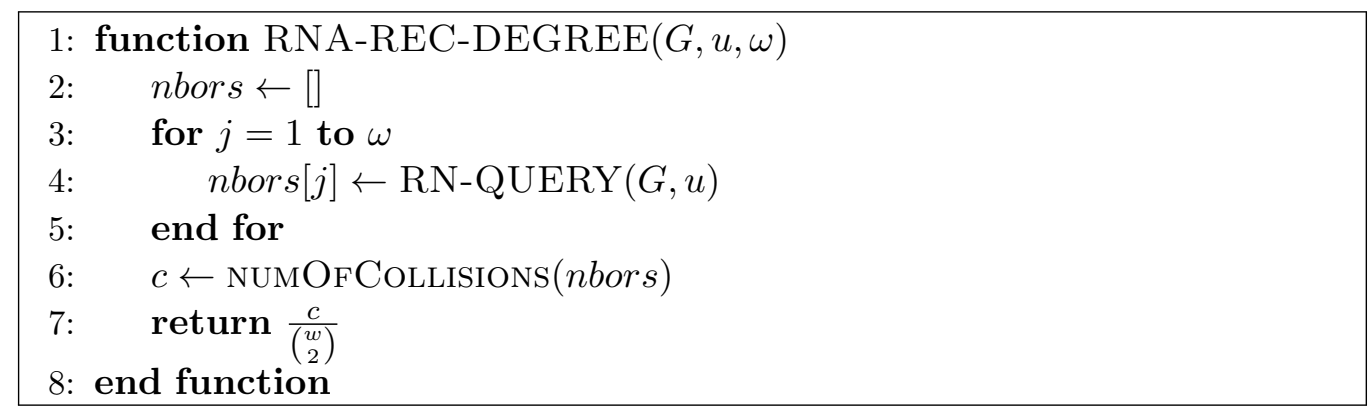

Algorithm 2: On-the-fly estimator for the reciprocal of the degree of the vertex $u$ under ERSRW with fixed probing.

Although we use fixed number of RN-QUERY calls for estimating the reciprocal of the degree of each sampled vertex, one important question in this context is how many RN-QUERY calls are needed to get a given precision and confidence level. Intuitively, a vertex with high degree requires more RNQUERY calls than a vertex with a small degree to get the same precision or confidence level. In the rest of this section, we develop the theory to 
establish the relation between the number of RN-QUERY calls and the degree for a given precision and confidence level. Note that an alternative approach might be to make variable number of RN-QUERY calls per vertex depending on the vertex degree. One possible approach is to make RN-QUERY calls until a fixed number of collisions occur. With this approach, we make more RN-QUERY calls for higher degree nodes. Our preliminary experimental results shows that this approach performs worse especially when the degree distribution is very skewed and sampling fraction is small. The main reason is that too many RN-QUERY calls are made for very high degree nodes. As a future work, we plan to further investigate under what circumstances this approach outperforms the fixed RN-QUERY per vertex approach.

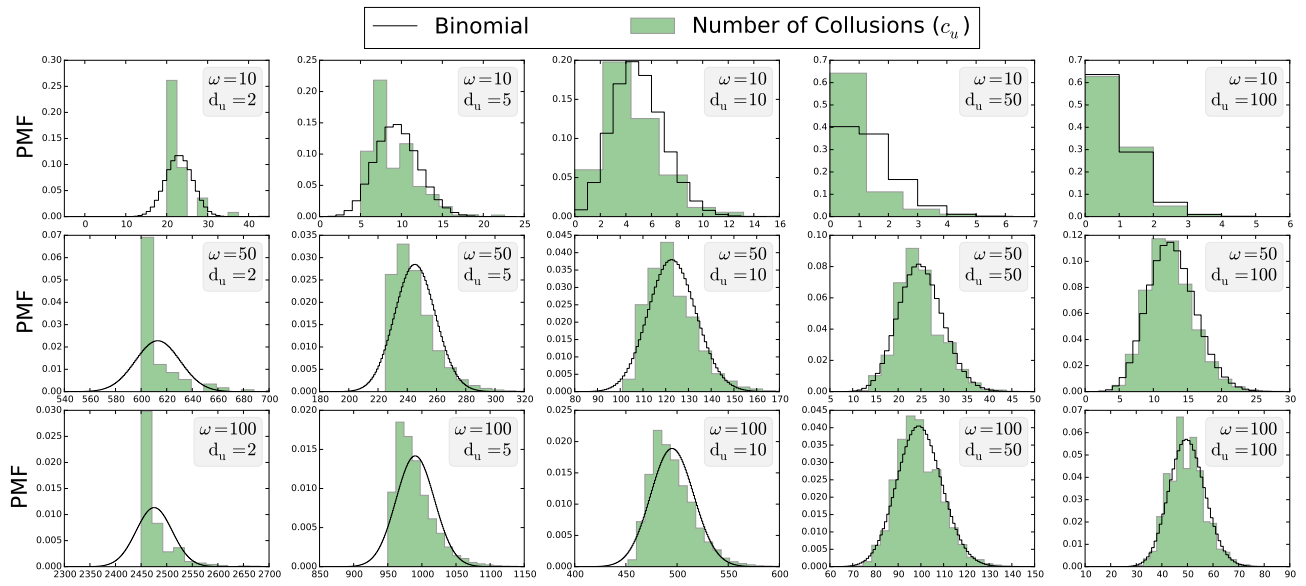

Figure 4: The accuracy of binomial approximation to the number of collisions $c_{u}$ for various $\omega$ and $d_{u}$ values. Each plot shows the normalized frequency of $c_{u}$ over 100,000 simulations (shown as filled-green bars) and the probability mass function (PMF) of $\operatorname{Binom}\left(\left(\begin{array}{c}\omega \\ 2\end{array}\right), 1 / d_{u}\right)$ (shown as black step lines).

We make the analysis for an arbitrary vertex $u$ without loss of generality. We use $c_{u}$ to denote the number of collisions in $\omega$ RN-QUERY calls for vertex $u$. We use the idea that $c_{u}$ follows approximately Binom $\left(\left(\begin{array}{c}\omega \\ 2\end{array}\right), 1 / d_{u}\right)$ as each ordered pair of sampled vertices have collision probability of $1 / d_{u} \cdot c_{u}$ is approximately binomial as the collisions of ordered pair of sampled vertices are not independent. Based on this approximation, we provide an estimator for $1 / d_{u}$ and a lower bound on $\omega$ for a given accuracy and precision in Theorem 1 . As the quality of the approximation in Theorem 1 depends on how well $c_{u}$ is approximated by $\operatorname{Binom}\left(\left(\begin{array}{c}\omega \\ 2\end{array}\right), 1 / d_{u}\right)$, we provide empirical results for various 
values of $\omega$ and $d_{u}$ in Fig 4 . The figure shows that binomial approximation is very good especially when both $\omega$ and $d_{u}$ are greater than 10 . Another observation is that although the approximations are not perfect for small $d_{u}$ (e.g. $d_{u}=2$ ), the mean and the variance of $c_{u}$ is still well approximated by $\operatorname{Binom}\left(\left(\begin{array}{c}\omega \\ 2\end{array}\right), 1 / d_{u}\right)$ (see Table 2). The main difference between them is the skewness. This helps us to use the variance of the binomial distribution as the approximate variance of the number of collisions and obtain approximate confidence intervals.

Theorem 1. The estimator $\hat{d}_{u}^{r e c}=\frac{c_{u}}{\left(\begin{array}{c}\omega \\ 2\end{array}\right)}$ for $1 / d_{u}$ is approximately unbiased and has mean square error $M S E\left(\hat{d}_{u}^{r e c}\right) \approx\left(\begin{array}{c}\omega \\ 2\end{array}\right)^{-1} \frac{1}{d_{u}}\left(1-\frac{1}{d_{u}}\right)$ and it guarantees for any $0 \leq \epsilon \leq 1$ and $0 \leq \alpha \leq 1$ :

$$
P\left[\left|\frac{\hat{d}_{u}^{r e c}-1 / d_{u}}{1 / d_{u}}\right| \leq \epsilon\right] \geq 1-\alpha
$$

when $\omega$ satisfies

$$
\omega \geq \frac{z_{\frac{\alpha}{2}} \sqrt{2\left(d_{u}-1\right)}}{\epsilon},
$$

where $z_{\alpha}$ denotes the 100(1- $\left.\alpha\right)$ th percentile of a standard normal distribution.

We first show the following two lemmas which are used to prove the Theorem 1:

Lemma 2. The estimator $\hat{d}_{u}^{r e c}$ is approximately unbiased for $1 / d_{u}$, i.e., $\operatorname{Bias}\left(\hat{d}_{u}^{r e c}\right) \approx 0$.

Proof. $c_{u}$ follows approximately a binomial distribution and $\left(\begin{array}{c}\omega \\ 2\end{array}\right)$ is a constant; therefore, $\mathbb{E}\left[\hat{d}_{u}^{r e c}\right]$ can be computed as:

$$
\mathbb{E}\left[\hat{d}_{u}^{r e c}\right]=\mathbb{E}\left[c_{u}\left(\begin{array}{l}
\omega \\
2
\end{array}\right)^{-1}\right]=\left(\begin{array}{c}
\omega \\
2
\end{array}\right)^{-1} \mathbb{E}\left[c_{u}\right] \approx\left(\begin{array}{l}
\omega \\
2
\end{array}\right)^{-1}\left(\begin{array}{l}
\omega \\
2
\end{array}\right) \frac{1}{d_{u}}=\frac{1}{d_{u}}
$$

Therefore, $\operatorname{Bias}\left(\hat{d}_{u}^{r e c}\right)=E\left[\hat{d}_{u}^{r e c}-1 / d_{u}\right] \approx 0$.

Lemma 3. The approximate variance of the estimator $\hat{d}_{u}^{r e c}, \operatorname{Var}\left(\hat{d}_{u}^{r e c}\right)$, is $\left(\begin{array}{c}\omega \\ 2\end{array}\right)^{-1} \frac{1}{d_{u}}\left(1-\frac{1}{d_{u}}\right)$ 
Table 1: Comparison of the estimators for SRW sampling with the adapted estimators for the RNA model. Estimators for the RNA model do not include the safety-margin dependency reduction technique to make it easier to compare with the estimators for SRW.

\begin{tabular}{ccc}
\hline Property Name & $\begin{array}{c}\text { Estimator for SRW sampling } \\
\text { (without dependency reduction) }\end{array}$ & $\begin{array}{c}\text { Estimator for te RNA model } \\
\text { (without dependency reduction) }\end{array}$ \\
\hline Average Degree & SRW-AVG-DEGREE & RNA-AVG-DEGREE \\
\hline & $\frac{n}{\sum_{u \in S} 1 / d_{u}}$ & $\frac{n}{\sum_{u \in S} 1 / d_{u}}$ \\
\hline \multirow{2}{*}{ Network Size } & SRW-VCB-NETSIZE & RNA-MVCB-NETSIZE \\
\cline { 2 - 3 } & $\frac{\sum_{u \in S} d_{u} \sum_{v \in S} 1 / d_{v}}{C}$ & $\frac{\sum_{u \in S} \Delta_{u} \sum_{v \in S} 1 / \Delta_{v}}{C}$ \\
& SRW-HHB-NETSIZE & RNA-HHB-NETSIZE \\
& $\frac{2 \hat{M}}{n} \sum_{u \in S} 1 / d_{u}$ & $\frac{2 \hat{M}}{n} \sum_{u \in S} \widehat{1 / d_{u}}$ \\
\hline
\end{tabular}

Table 2: Comparison of the descriptive statistics (mean, variance, and skewness) of $\operatorname{Binomial}\left(\left(\begin{array}{c}\omega \\ 2\end{array}\right), 1 / d_{u}\right)$ and the number of collisions $c_{u}$ for various $\omega=\{10,50,100\}$ and $d_{u}=\{2,5,10,5,100\}$.

\begin{tabular}{|c|c|c|c|c|c|c|c|c|c|}
\hline & \multicolumn{3}{|c|}{$\omega=10$} & \multicolumn{3}{|c|}{$\omega=50$} & \multicolumn{3}{|c|}{$\omega=100$} \\
\hline & mean & var & skew & mean & var & skew & mean & var & skew \\
\hline \multicolumn{10}{|l|}{$d_{u}=2$} \\
\hline Binomial & 22.500 & 11.250 & 0 & 612.500 & 306.250 & 0 & $2,475.000$ & $1,237.500$ & 0 \\
\hline$c_{u}$ & 22.529 & 11.434 & 2.367 & 612.458 & 303.849 & 2.755 & $2,474.968$ & $1,247.998$ & 2.847 \\
\hline \multicolumn{10}{|l|}{$d_{u}=5$} \\
\hline Binomial & 9.000 & 7.200 & 0.224 & 245.000 & 196.000 & 0.043 & 990.000 & 792.000 & 0.021 \\
\hline$c_{u}$ & 9.010 & 7.251 & 1.423 & 245.059 & 196.995 & 1.392 & 990.004 & 798.642 & 1.421 \\
\hline \multicolumn{10}{|l|}{$d_{u}=10$} \\
\hline Binomial & 4.500 & 4.050 & 0.398 & 122.500 & 110.250 & 0.076 & 495.000 & 445.500 & 0.038 \\
\hline$c_{u}$ & 4.504 & 4.064 & 1.219 & 122.507 & 110.369 & 1.010 & 494.982 & 443.776 & 0.967 \\
\hline \multicolumn{10}{|l|}{$d_{u}=50$} \\
\hline Binomial & 0.900 & 0.882 & 1.022 & 24.500 & 24.010 & 0.196 & 99.000 & 97.020 & 0.097 \\
\hline$c_{u}$ & 0.897 & 0.875 & 0.377 & 24.503 & 23.988 & 0.574 & 99.013 & 97.040 & 0.491 \\
\hline \multicolumn{10}{|l|}{$d_{u}=100$} \\
\hline Binomial & 0.450 & 0.445 & 1.468 & 12.250 & 12.128 & 0.281 & 49.500 & 49.005 & 0.140 \\
\hline$c_{u}$ & 0.446 & 0.436 & 1.645 & 12.244 & 12.177 & 0.577 & 49.531 & 49.278 & 0.427 \\
\hline
\end{tabular}


Proof. The result simply follows from the fact that $\omega$ is a constant and $c_{u}$ follows approximately $\operatorname{Binom}\left(\left(\begin{array}{c}\omega \\ 2\end{array}\right), 1 / d_{u}\right)$.

Proof of Theorem 1. In Lemma 2, we show that $\hat{d}_{u}^{\text {rec }}$ is approximately unbiased for $1 / d_{u}$. Then, in Lemma 3 , we obtain the variance of $\hat{d}_{u_{a}}^{r e c}$ which is approximately $\operatorname{MSE}\left(\hat{d}_{u}^{r e c}\right)$ as $M S E\left(\hat{d}_{u}^{r e c}\right)=\operatorname{Var}\left(\hat{d}_{u}^{r e c}\right)+\operatorname{Bias}^{2}\left(\hat{d}_{u}^{r e c}\right)$ and $\operatorname{Bias}^{2}\left(\hat{d}_{u}^{r e c}\right) \approx 0$. In order to show the lower bound for $\omega$, we use the normal distribution approximation to the binomial distribution. We use Normal(mean, variance) to denote the normal distribution with a given mean and variance. As a rule of thumb, a binomial distribution $\operatorname{Binom}(n, p)$ is well-approximated by a normal distribution $\operatorname{Normal}(n p, n p(1-p))$ when $n p>5$ and $n(1-p)>5$. Therefore, $\hat{d}_{u}^{r e c}$ can be approximated by the $\operatorname{Normal}\left(1 / d_{u},\left(\begin{array}{c}\omega \\ 2\end{array}\right)^{-1} \frac{1}{d_{u}}\left(1-\frac{1}{d_{u}}\right)\right)$ when $\left(\begin{array}{c}\omega \\ 2\end{array}\right) / d_{u}>5$ and $\left(\begin{array}{c}\omega \\ 2\end{array}\right)\left(1-\frac{1}{d_{u}}\right)>5$, or approximately when $\omega>2 \sqrt{10 d_{u}}$. Note that for reasonable $\epsilon(<0.1)$ and $z_{\frac{\alpha}{2}}(>1.64)$ values, the lower bound for $\omega$ in Theorem 1 is tighter. Therefore, normality assumption is safe to make. Next, by combining the normal approximation and setting the desired margin of error to $\frac{\epsilon}{d_{u}}$, we get the lower bound on $\omega$ as:

$$
\omega \geq \frac{1+\sqrt{1+\frac{8 z_{\alpha / 2}^{2}\left(d_{u}-1\right)}{\epsilon^{2}}}}{2} \approx \frac{\sqrt{\frac{8 z_{\alpha / 2}^{2}\left(d_{u}-1\right)}{\epsilon^{2}}}}{2}=\frac{z_{\frac{\alpha}{2}} \sqrt{2\left(d_{u}-1\right)}}{\epsilon} .
$$

Fig. 5a and 5b illustrate the relation between degree, precision, and confidence level obtained in Eq. 10. Fig. 5a illustrates the required number of RN-QUERY calls to get an estimate within a desired relative error with $90 \%$ confidence level. Fig. 5b illustrates the required number of API calls to get an estimate with a desired confidence level within $\epsilon=0.1$ relative error. Fig. 6 illustrates the mean and coefficient of variance of 10,000 independent degree estimations using various number of RN-QUERY calls for different vertex degrees and the normalized root mean square error (NRMSE) where the normalization is over the true parameter, i.e., $\operatorname{NRMSE}(\hat{\theta})=E[(\hat{\theta}-\theta)]^{1 / 2} / \theta$. As the vertex degree increases, negative bias is observed for small $\omega$. This

\footnotetext{
${ }^{7} 1 / d_{u} \leq\left(1-1 / d_{u}\right)$ for $d_{u} \geq 2$. Therefore, the bound is obtained using $\left(\begin{array}{c}\omega \\ 2\end{array}\right) / d_{u}>5$. We handle $d_{u}=1$ case separately We estimate $1 / d_{u}$ as 1 when all RN-QUERY calls return the same node.
} 

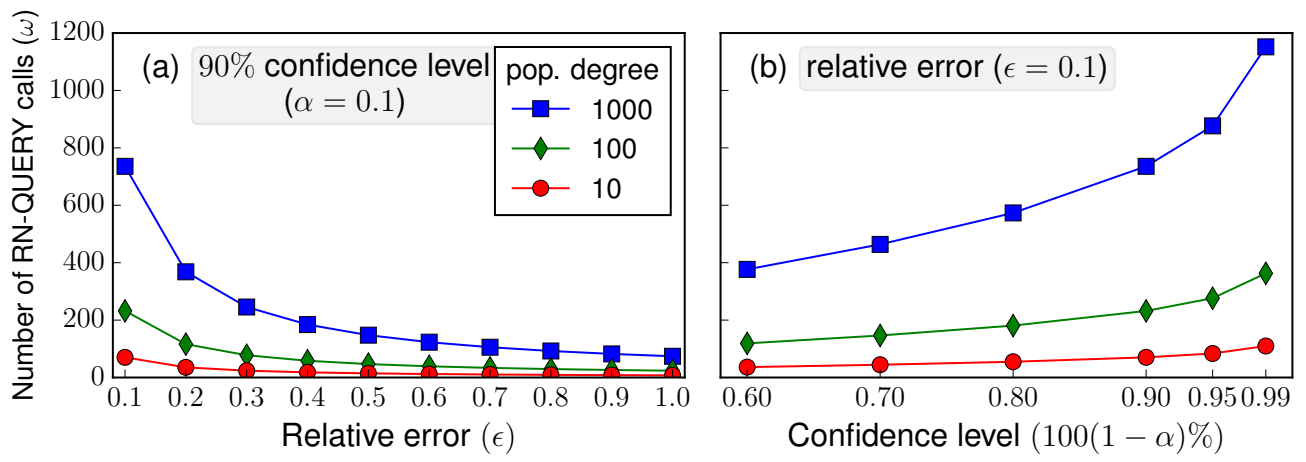

Figure 5: Relation between number of RN-QUERY calls and degree for a given confidence level and relative error. (a) Relative error vs. the minimum $\omega$ needed for $90 \%$ confidence level (b) Confidence level vs. the minimum $\omega$ needed for the relative error $\epsilon=0.1$.

observation can be explained by the high likelihood of no collision when $\omega$ is much less than the population degree of a vertex. When there is no collision, the estimator becomes 0 . Fig. 6 also shows the NRMSE of 10,000 estimations for various degrees with varying $\omega$. As expected, we get a higher NRMSE for the estimation of a higher degree with the same $\omega$.

\subsection{Average Degree Estimator : RNA-AVG-DEGREE}

In Section 2.4.1, we discussed SRW-AVG-DEGREE, a GENERALIZED estimator with shifted-thinning. Although SRW-AVG-DEGREE is a wellknown estimator under SRW sampling, it can not be directly used to estimate the average degree as the degrees of sampled vertices are not observable under the RNA model. In this section, we introduce RNA-AVG-DEGREE estimator that is a simple adaptation of SRW-AVG-DEGREE estimator to the RNA model by estimating the reciprocal of the vertex degrees using RNAREC-DEGREE. The pseudocode of the RNA-AVG-DEGREE is shown in Algorithm 3. In line 2, we first determine $n_{\omega}$, the number of nodes visited by the walk. The algorithm can also be implemented in such a way that the it stops when the sampling budget $n$ is reached. In line 3 , we initiate the list recTotal which keeps the sum of the reciprocal of the vertex degree estimates for each $t$ thinned walk. Recall that shifted-thinning with parameter $t$ creates $t$ thinned walks, where thinned walk $i \in[1, t]$ consists of vertices in $\left\{s_{i+0 t}, s_{i+1 t}, \ldots\right\}$. Then, between lines 5-9, we perform ERSRW and compute the sum of the estimates of reciprocal of the vertex degrees for each thinned walk. Note that we use line 9 for ease of presentation. It can be 

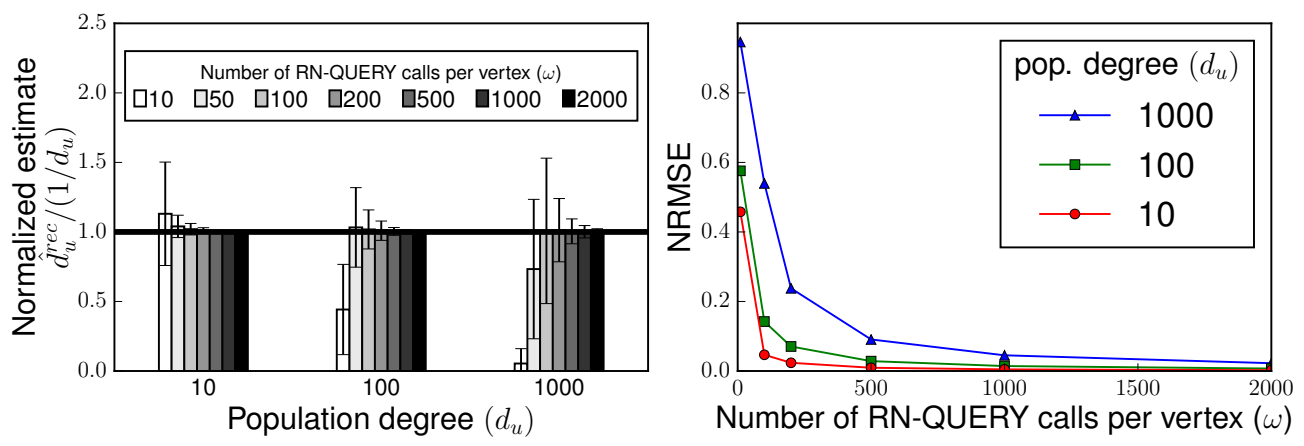

Figure 6: Accuracy of each bar group shows how the number of RN-QUERY calls affects the degree estimation performance and precision. Bar lengths and error bars represent the mean and coefficient of variation of 10,000 independent degree estimations, respectively. $y$-axis is normalized by the population degree.

eliminated by using one of the RN-QUERY calls in RNA-REC-DEGREE at each iteration. In our implementation, we use the last RN-QUERY calls in RNA-REC-DEGREE to determine next step in the walk. Finally, on line 10, the average of the $t$ estimates based on $t$ thinned walks is computed.

Under a fixed sampling budget, there is a tradeoff between the estimation performance of vertex degrees and number of vertices visited by the walk as the number of vertices in the walk will be approximately $n / \omega$. The larger the $\omega$ is, the better the vertex degree estimations are, but the smaller the length of the walk. We explore this tradeoff using simulations in Section 5.3.

\subsection{Network Size Estimators}

In this section we propose two different estimators for the network size: 1) Multiplicity and Vertex Collision Based Network Size (RNA-MVCB-NETSIZE) Estimator and 2) Hansen-Hurwitz-based Network Size (RNA-HHB-NETSIZE) Estimator.

\subsubsection{RNA-MVCB-NETSIZE Estimator}

In this section, we propose a network size estimator under the RNA model by adapting the SRW-VCB-NETSIZE estimator using the well-known Ergodic Theorem in the Markov chain theory (see Appendix A). The estimator has two requirements: (a) vertices need to be sampled from the stationary distribution of the underlying graph independently and (b) vertex degrees need to be known or estimated up to a constant. We use a stationary RSRW 


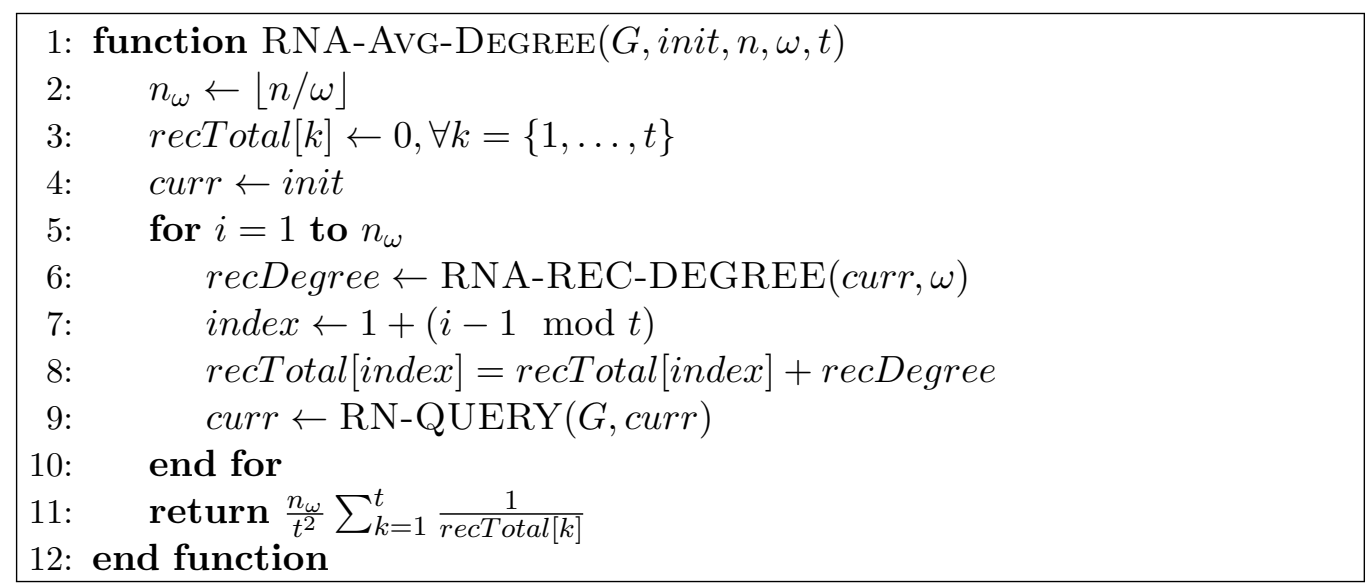

Algorithm 3: Average degree estimator for the RNA model.

and apply the safety-margin dependency reduction technique proposed in [6] to meet the first requirement. We estimate the vertex degrees up to a constant using the vertex multiplicities based on the Ergodic Theorem to meet the second requirement.
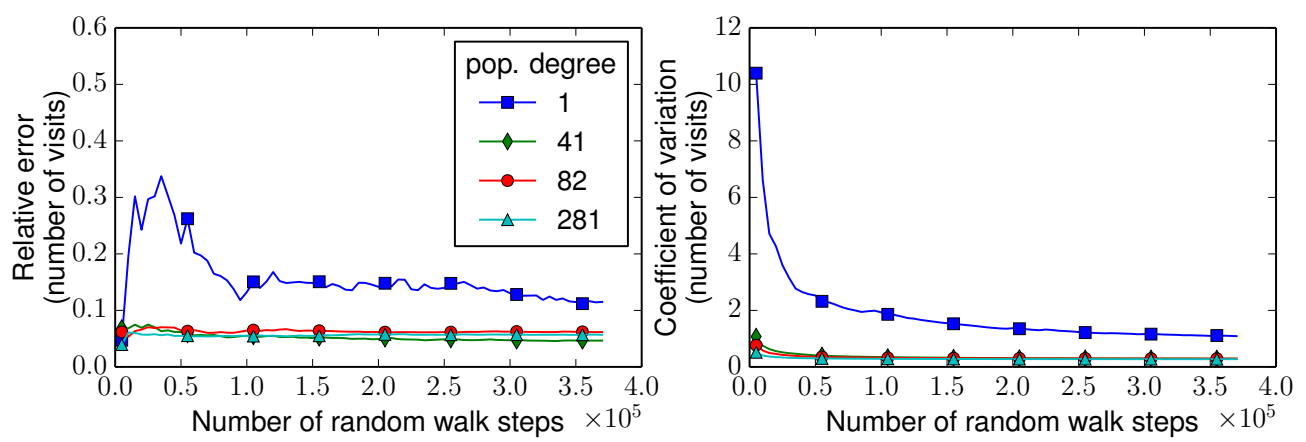

Figure 7: Relative error and coefficient of variation of number of visits to four vertices with different degrees in CONDMAT graph. Each point is calculated based on 1,000 independent simulations.

Theorem 4 simply states that the multiplicity of a vertex becomes proportional to its degree in the limit of a large sample size. Although the multiplicity of a vertex $(\Delta)$ is not guaranteed to be proportional to the degree of that vertex for small sample sizes, it is still a reasonable characteristic to consider under the RSRW which a very limited sampling design. Therefore, we use the multiplicity of a vertex in Algorithm 1 in place of the vertex 
degree. In order to illustrate how $\Delta(i, t)$ converges to its theoretical value for finite sample sizes, we conduct an experiment on CONDMAT real world graph (see Table 3 for the details of the graph). The results are shown in Fig. 7. We select four nodes with different degrees. We perform 1,000 independent random walks of length 400,000. For each walk, we keep track of how many times each of these four vertices is visited after every 5,000 steps. Finally, we find the relative error with respect to the right hand side of Eq. A.1 in the Ergodic Theorem and plot the average of the 1,000 relative errors. We observe that the relative error and the coefficient of variation is small for vertices with larger degrees even for the initial steps of the RW. We want to emphasize that the convergence results in Fig. 7 are specific to CONDMAT graph. In general, the convergence of a random walk is a wellstudied topic and it has been shown that it depends on the "spectral gap" of the underlying graph which is defined as $1-\lambda_{2}$ where $\lambda_{2}$ is the second largest eigenvalue of the transition matrix of the underlying graph[28].

The pseudocode of the network size estimator using vertex multiplicities is given in Algorithm 4. Between lines 3-10, the multiplicity of sampled nodes are computed. Between lines 12-21, the estimator is computed using the multiplicities of the sampled nodes. On line 14, the algorithm checks whether the samples are apart from each other in the walk for the dependency reduction. If it is true, than the RNA-MVCB-NETSIZE estimator as shown in Table 1 is computed. Recall that the estimators in Table 1 do not include the dependency reduction techniques for the sake of clear comparison. Although the proposed algorithm has $O\left(n^{2}\right)$ running time complexity, we present this version due to its readability. The complexity can easily be reduced to $O(n)$ by using a similar idea as in [6].

\subsubsection{RNA-HHB-NETSIZE Estimator}

In this section, we propose another estimator named RNA-HHB-NETSIZE for the network size. The basic idea is as follows: we have proposed an estimator for the average degree $\mu_{d}$ under ERSRW sampling in Section 4.2. If we can develop an estimator for the number of edges $M$ under the same sampling scheme, then the network size $N=2 M / \mu_{d}$ can be estimated.

At first, estimating $M$ seems to be as hard as estimating $N$. However, obtaining edges uniformly at random is easier and more efficient than obtaining a vertex uniformly at random under ERSRW sampling. Note that we make RN-QUERY calls for two reasons under ERSRW sampling. First, we make $\omega$ RN-QUERY calls for the same vertex to estimate the reciprocal 


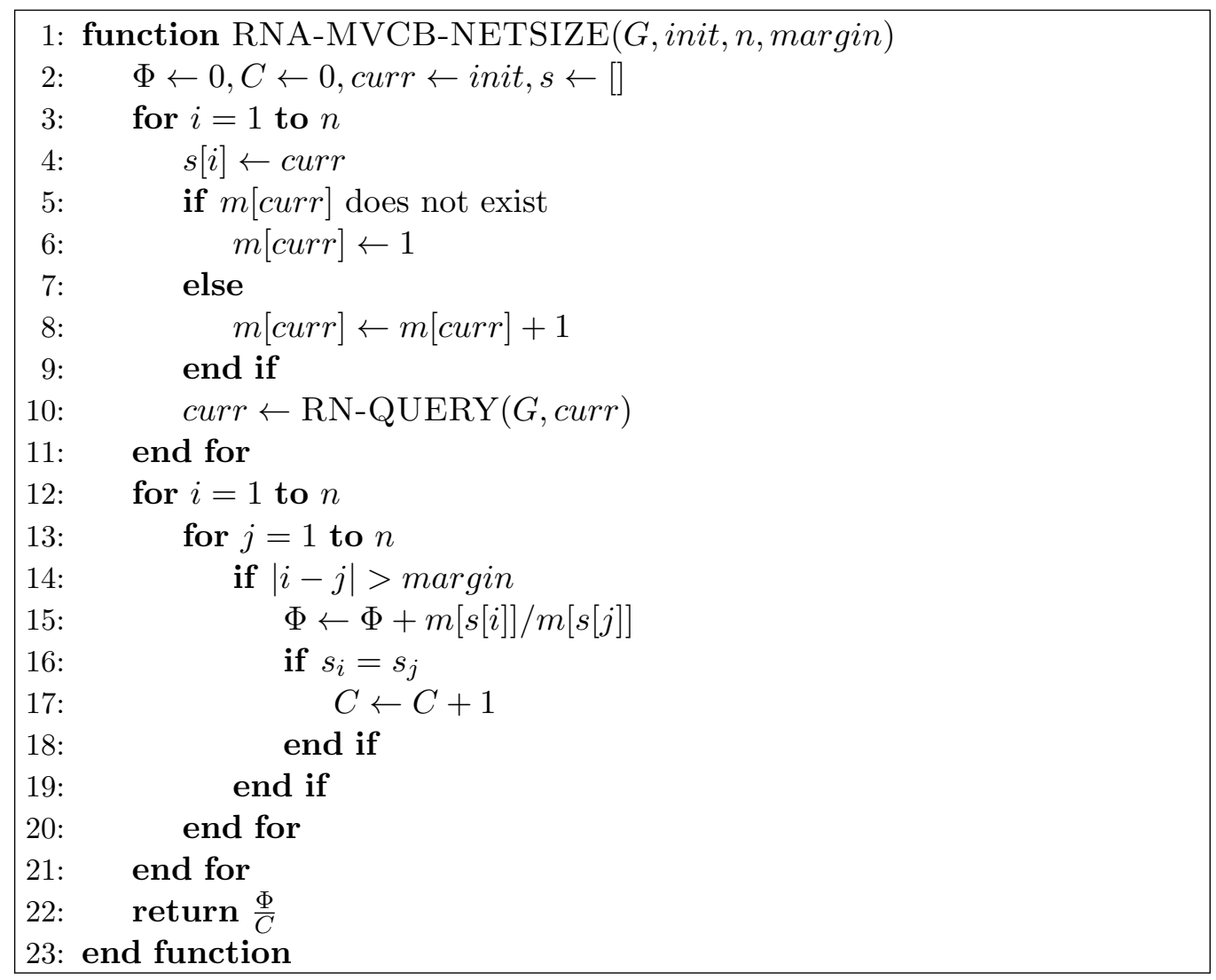

Algorithm 4: Multiplicity and vertex-collision based network size estimator for the RNA model.

of the vertex degree. Second, we use the last RN-QUERY call to go to a random neighbor, i.e., to perform a walk on the graph. If we consider the RN-QUERY calls made for the walk and take the current vertex and randomly selected neighbor vertex tuples as a sample edge at each step, we can sample the edges uniformly in the long run. Then, the challenge is to estimate the number of edges when we can sample the edges uniformly. This is a very similar problem to network size estimation, but the sampled objects are edges instead of nodes and they are selected with equal probability. Using Katzir's estimator with weights equal to 1 (hence both $\Phi$ and $\Psi$ becomes $\left.n_{\omega}\right)$, we get the following estimator for $M: \hat{M}=\frac{n_{\omega}^{2}}{2 C_{E}}$, where $C_{E}$ is the number of collisions in the sampled edges. Then, by applying the safety-margin 


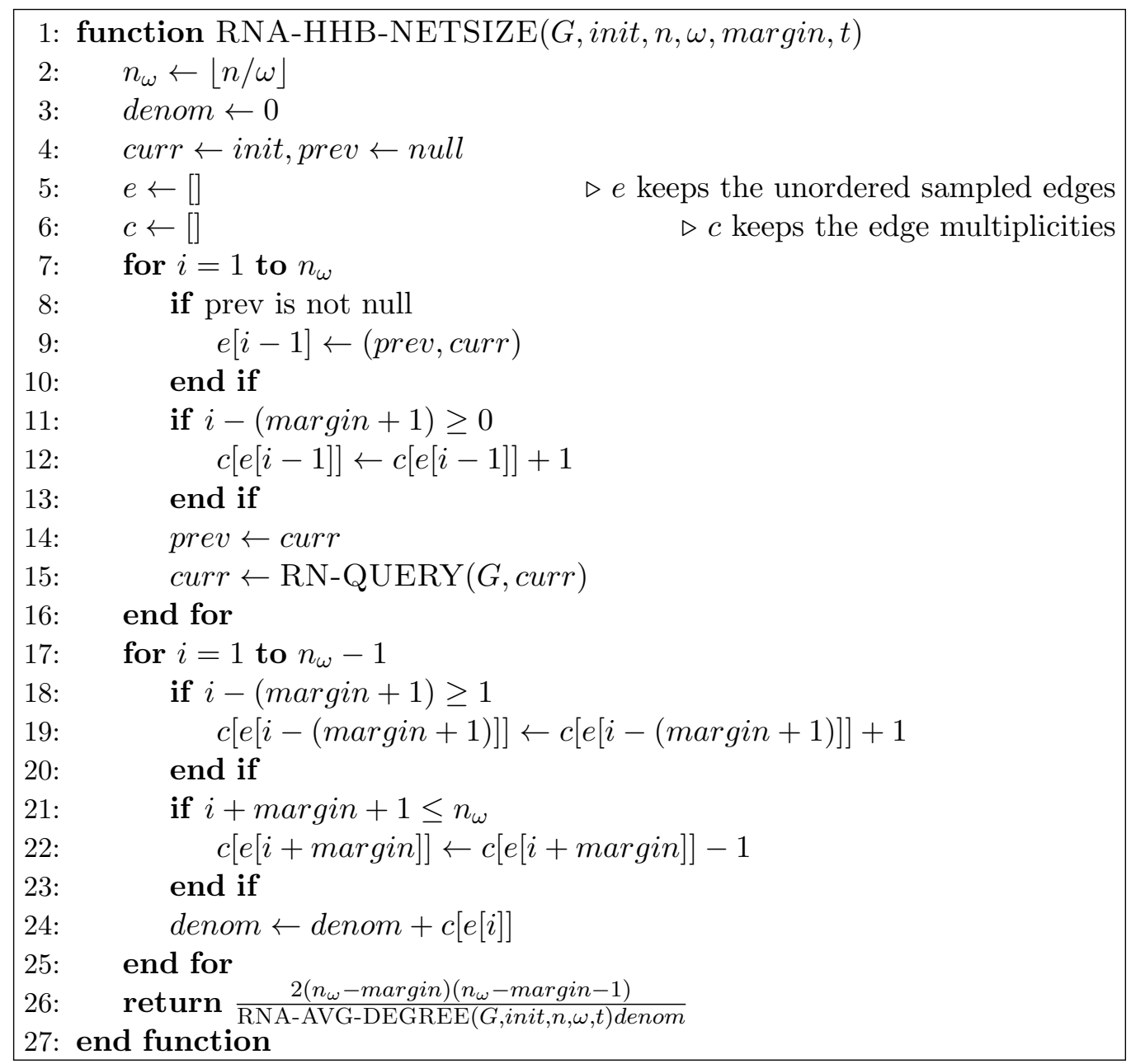

Algorithm 5: Hansen-Hurwitz-based network size estimator for the RNA model.

technique, we get

$$
\hat{M}=\frac{\sum_{1 \leq i, j \leq n_{\omega}} \mathbf{1}_{\{|j-i|>\text { margin }\}}}{\sum_{1 \leq i, j \leq n_{\omega}} \mathbf{1}_{\left\{s_{e_{i}}=s_{e_{j}}\right\}} \mathbf{1}_{\{|j-i|>\text { margin }\}}}=\frac{\left(n_{\omega}-\operatorname{margin}\right)\left(n_{\omega}-\operatorname{margin}-1\right)}{\sum_{1 \leq i, j \leq n_{\omega}} \mathbf{1}_{\left\{s_{e_{i}}=s_{e_{j}}\right\}} \mathbf{1}_{\{|j-i|>\text { margin }\}}},
$$

where $s_{e_{i}}$ denotes the $i^{\text {th }}$ sampled edge. Finally, dividing $2 \hat{M}$ by RNA-AVGDEGREE estimator proposed in Section 4.2, we get the estimator for $N$. The pseudocode of the efficient implementation is given in Algorithm 5. In 
lines 8-10, sampled unordered edges 8 are stored in list e. In lines 11-13, multiplicities of sampled edges are computed. $c[e[i]]$ denotes the multiplicity of $i^{\text {th }}$ sampled edge. In lines 17-25, twice the total number of edge collisions that are at least margin step away from each other, i.e., the denominator of Eq. 11, is computed and stored in denom ${ }^{9}$ Finally, in line 26, estimation for the average degree and the number of edges is combined to get the estimation for the network size. For ease of presentation, we compute the RNA-AVGDEGREE estimator in line 26, but it can also be computed in the first for loop of the algorithm (between lines 7-16).

\section{Experimental Results}

In this section we evaluate the performance of the proposed estimators. In general, comparing two estimators under a different data access model may not be fair as one of the models may allow more access to the data for a given estimation task and this may make the estimation problem easier. For this reason, we do not compare the performance of our estimators with the estimators developed under a different sampling design such as independent vertex/edge sampling or induced subgraph sampling. Instead, we compare the proposed estimators with the corresponding SRW based estimators in which vertex degrees are observed (see Table 1). Comparing the performance of the SRW and RNA based estimators enables us to observe the two phenomena: 1) the performance of the estimators with the true degree information of the sampled vertices and 2) the performance drop due to the estimation of the vertex degrees in the RNA based estimators.

\subsection{Dataset}

We evaluate the performance of our estimators on both real-world networks from various application domains and synthetic networks.

Real-World Networks: The real-world network datasets that we use in the experiments are shown in Table 3. We extract and use only the largest connected component of each graph. The description of the real world-graphs are as follows:

\footnotetext{
${ }^{8}$ Edge $(u, v)$ and edge $(v, u)$ is considered to be the same.

${ }^{9}$ Collision on indices $(i, j)$ and $(j, i)$ are counted separately.
} 
Table 3: Details of real-world networks used in the experiments. All except Facebook (New-Orleans) network was obtained from [76].

\begin{tabular}{|c|c|c|c|c|}
\hline Network Name & \# of nodes in LCC & \# of edges in LCC & avg. degree & $\begin{array}{c}\text { degree assortativity } \\
\text { coefficient (r) }\end{array}$ \\
\hline GNUTELLA & $62,561\left(1.000^{*}\right)$ & $147,878\left(1.000^{*}\right)$ & 4.727 & -0.09 \\
\hline CONDMAT & $21,363(0.923)$ & $91,342(0.977)$ & 8.551 & 0.13 \\
\hline EPINIONS & $75,877(1.000)$ & $508,836(1.000)$ & 10.695 & -0.04 \\
\hline ENRON & $33,696(0.918)$ & $180,811(0.984)$ & 10.732 & -0.12 \\
\hline FACEBOOK [77] & $63,392(0.995)$ & $816,886(1.000)$ & 25.773 & 0.18 \\
\hline
\end{tabular}

$*$ the fraction of the number of nodes in the largest connected component (LCC)

$* *$ the fraction of the number of edges in the LCC

- GNUTELLA: Gnutella peer-to-peer file sharing network from August 2002. Vertices are hosts in Gnutella network and there exists an edge between two vertices if they have connections.

- CONDMAT: Condense Matter Physics collaboration network. It covers scientific collaborations between authors papers submitted to Condense Matter category in arXiv.org between January 1993 and April 2003.

- EPINIONS: Who-trusts-whom network of users of Epinions.com. Although the trust relationship is directed in the original data set, we convert it into an undirected graph where there exists an edge between two vertices if there exists at least one directed edge between them for the sake of analysis.

- ENRON: Enron email communication network. Vertices are the email addresses and there exists an undirected edge between two vertices if there is at least one email communication between them.

- FACEBOOK: New Orleans Regional Facebook friendship network collected between December 29, 2008 and January 3, 2009 in a breadthfirst-search fashion.

Synthetic Networks: We use two synthetic graph generation models: 1) Erdős-Renyi (ER) and 2) Barabási-Albert (BA). In the experiments, we generate synthetic networks of size 10,000 with average degrees 4, 10, and 100 .

ER graph generation model creates purely random networks (we shortly refer to them as ER graphs) and has two general forms. In one, first the 
number of edges and vertices are fixed, then these edges connect randomly selected vertices. In the other, which we use in this paper, an edge is created between each pair of vertices with probability $p$. One can create ER graphs with size $N$ and average degree $\mu_{d}$ by setting the $p$ value to $\frac{\mu_{d}}{N-1}$ as the expected number of edges in the generated network is $\left(\begin{array}{c}N \\ 2\end{array}\right) p$.

BA graph generation model creates scale-free networks (we shortly refer to them as BA graphs) and the model incorporates two important concepts: 1) preferential attachment and 2) growth. Growth means that the model creates a network with increasing size over time. Preferential attachment means that the vertices with higher degree have more chance to connect to the new nodes. These two concepts are very common in real-world networks. One can create BA graphs with size $N$ and average degree $\mu_{d}$ by setting the parameter for the number of edges attached to the newly joining node to $\mu_{d} / 2$. The reason is that the number of edges in the network converges to $N \mu_{d} / 2$ as $N \rightarrow \infty$ and hence $\mu_{d}$ is obtained as the approximate average degree.

Both ER and BA graph generation models generate non-assortative graphs in the limit of large graph size. In other words, the degree of a node is not correlated with the degrees of its neighbors. This is an important feature as we perform a random walk on the underlying graph, we know that the vertex degrees of sampled nodes are not correlated. Therefore, the effect of correlation in the degrees of sampled nodes disappear on the estimation performance. This helps us eliminate one possible side effect on the estimation performance as we generate graphs with different parameters.

\subsection{Evaluation Metric}

We evaluate the performance of the proposed estimators using simulations as follows: We collect 1,000 independent samples of a certain size. Then, we use the relevant estimator to create 1,000 independent estimates of a certain property. Then, we compute the $5^{\text {th }}$ and $95^{\text {th }}$ percentile of these 1,000 estimations. The region between these two intervals is known as $90 \%$ Monte Carlo confidence interval. We also compute the $50^{\text {th }}$ percentile (median) of these 1,000 estimations. Then, we normalize these percentile values with the true parameter to illustrate how well the estimations are concentrated around the true parameter. We repeat this experiment for various sampling fractions. For the average degree, we use the sampling fraction range of $[0.01,1]$. For the network size, we use the sampling fraction range of $[0.01,50]$. If the 
region between $5^{\text {th }}$ and $95^{\text {th }}$ percentiles concentrates around 1 , the estimation performance gets better.

\subsection{Average Degree Estimation}

We compare the RNA-AVG-DEGREE estimator with the SRW-AVGDEGREE estimator. In the RNA model, it is not possible to get the vertex degree with $100 \%$ certainty. Therefore, we need to determine a fair cost function for obtaining the actual vertex degree in the SRW-AVG-DEGREE estimator. One approach could be that a single RN-QUERY call returns the actual degree of a vertex as well as its id. However, this assumption will trivially make SRW-AVG-DEGREE estimator better because we use $\omega$ RN-QUERY calls in the RNA model in order to estimate the vertex degree. Instead, we use the same walk length (of size $\lfloor n / \omega\rfloor$ ) for both estimators. This corresponds to getting the actual vertex degree with a sampling cost of $\omega$ in SRW-AVG-DEGREE estimator. With this approach, we can observe the effect of degree estimations on the performance of the average degree estimation by the difference of SRW-AVG-DEGREE and RNA-AVG-DEGREE confidence intervals.

The summary of results for the average degree estimation is as follows

- The estimation precision depends on the degree structure of the underlying graph (e.g., average degree, degree variance, degree skewness), the value of $\omega$, and the sampling fraction.

- A good selection of $\omega$ depends on the degree structure of the underlying graph. When the degree variance is not high and the sampling fraction is small, optimal value of

- Applying shifted-thinning dependency technique to the walk decreases estimation performance.

Real-world graphs: The results for real-networks with no thinning, $t=1$, is shown in Fig. 8. We first note that the confidence intervals for SRW-AVGDEGREE estimator get wider as $\omega$ increases. This is because the length of SRW $n / \omega$ decreases and the shorter walk length causes less precise estimations. We also observe that the confidence intervals for SRW-AVG-DEGREE and RNA-AVG-DEGREE overlaps. This is probably because $\omega=10$ is large enough for the degree estimations which can be validated by the small true average degrees of the population graphs shown in Table 3. As we will show 
in the synthetic graph cases, when the true average degree is high (e.g., 100), RNA-AVG-DEGREE performs worse than SRW-AVG-DEGREE for $\omega=10$.

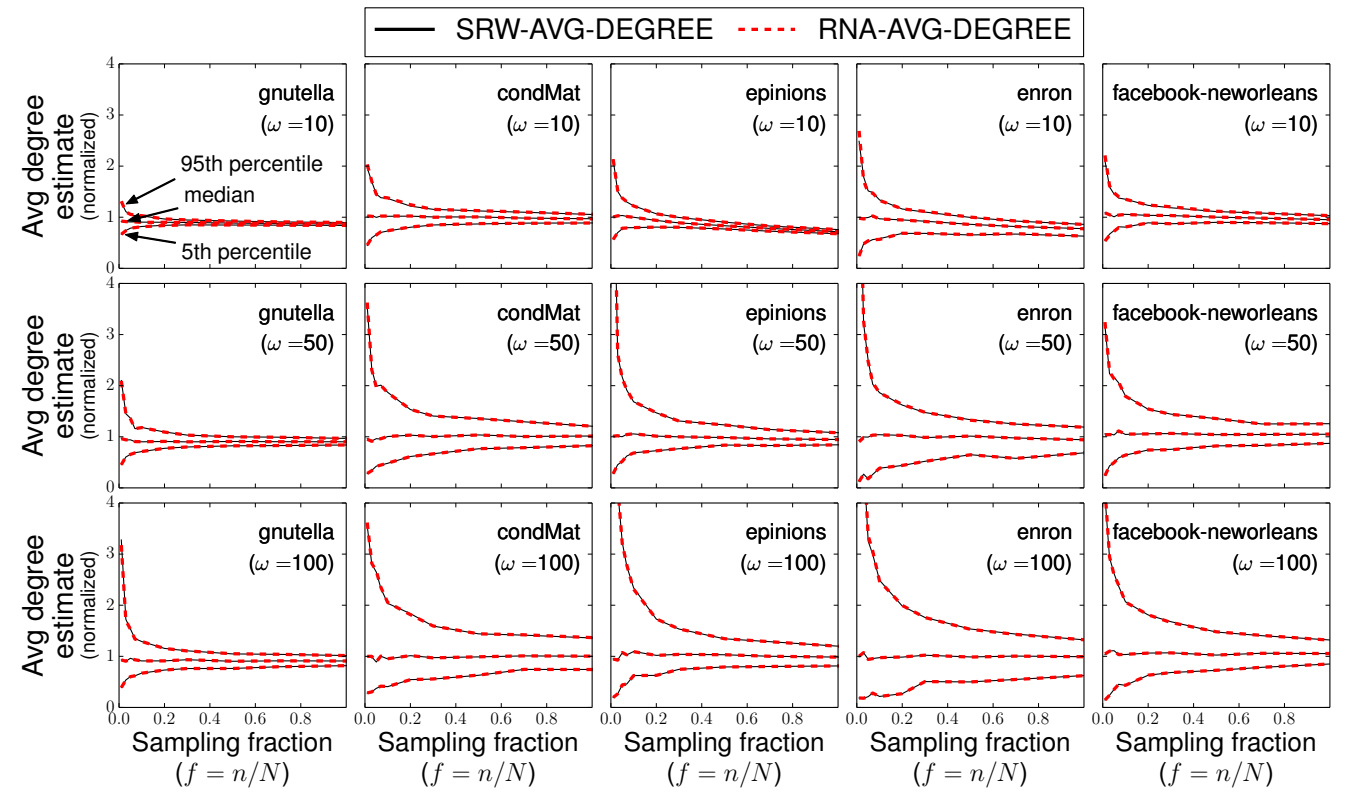

Figure 8: Average degree estimation (real-world graphs) with no thinning, $\mathbf{t}=1$ : $5^{\text {th }}$ percentile, median, and $95^{\text {th }}$ percentile of 1,000 estimations are shown for both SRWAVG-DEGREE and RNA-AVG-DEGREE estimators. Estimation results for the sampling fractions ranging from $1 \%$ to $100 \%$ are shown on each plot. Estimation results with three different $\omega$ values are shown on each row: $\omega=[10,50,100]$.

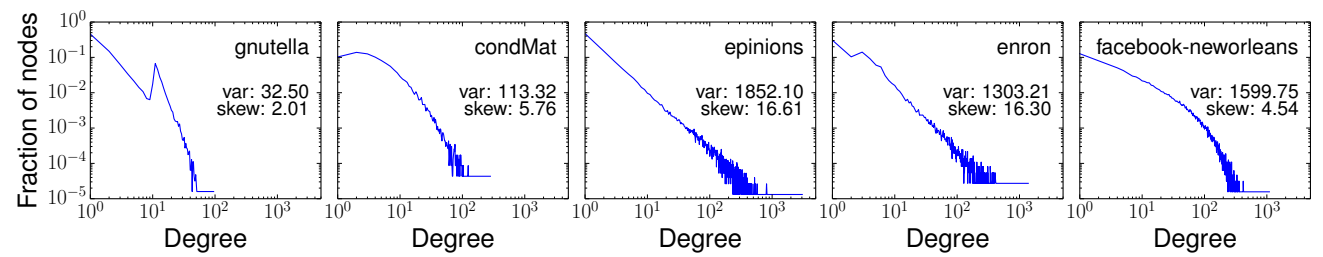

Figure 9: Degree distributions and statistics of the real-world graphs. var and skew stand for the variance and the skewness of the degree distribution, respectively.

Another observation is that the estimation in GNUTELLA graph is much better than all others for all $\omega$ that we consider. An important reason might be that GNUTELLA graph has a lower average degree, degree variance, and skewness (see Fig 9). An interesting observation is that a small downward bias is observed when $\omega=10$ and $f>0.8$, especially in ENRON and EPIN- 
IONS graphs. This might be due to high variance and skewness in the degree distribution. Approximately $85 \%$ of the vertices have degrees less than the true average degree in ENRON and EPINIONS graphs; while approximately $70 \%$ of the vertices have degrees less than the true average degree in the other real-world graphs. Nevertheless, we should not observe this downward bias if we repeat the experiments infinitely many times. The same phenomena occurs when estimating the mean of a highly skewed distribution with i.i.d. samples. If the sample size is not large enough, the sampling distribution is also skewed. Furthermore, the more skewed the distribution is, the larger sample size we need to get a normally distributed sampling distribution. We also observe that high degree variance with a small walk length causes a very wide confidence intervals for small $f<0.1$. For instance, see ENRON, EPINIONS, and FACEBOOK graphs with $\omega=100$.

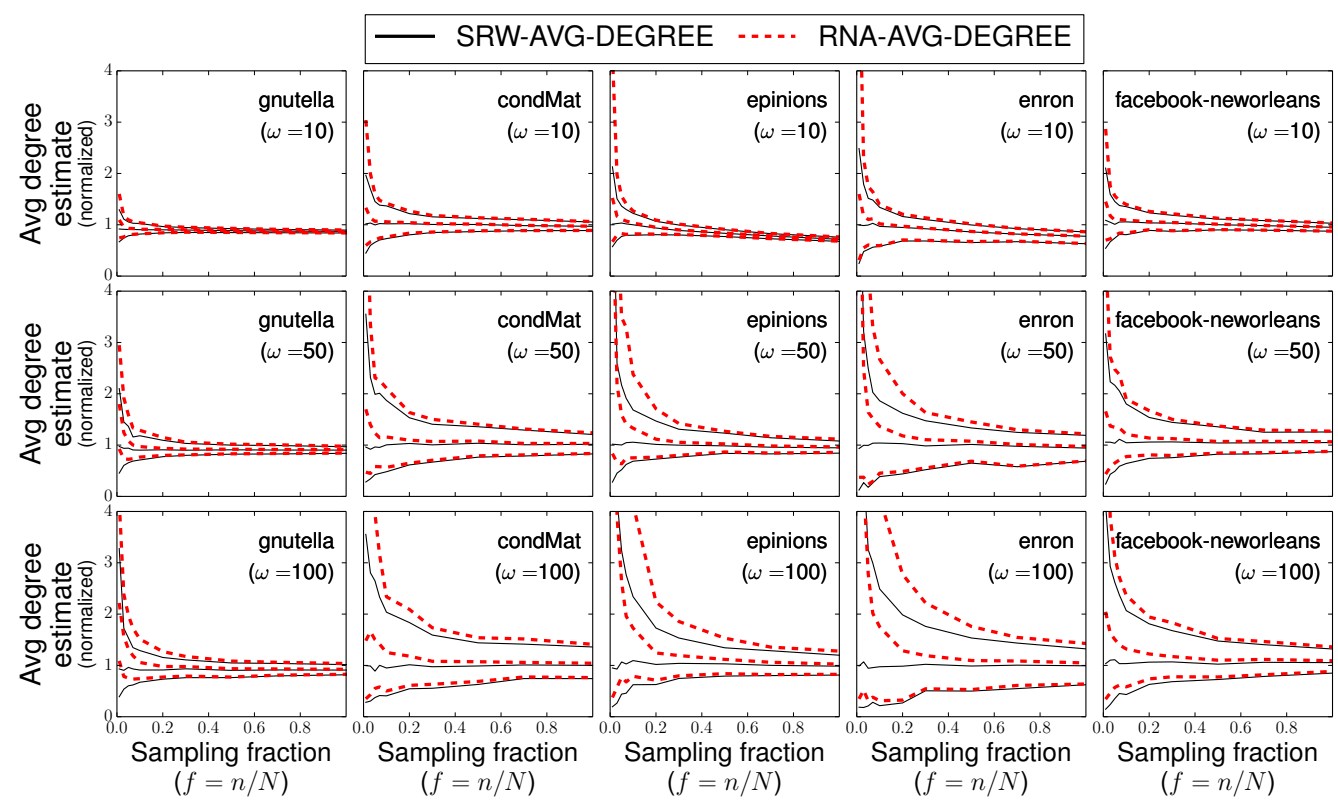

Figure 10: Average degree estimation (real-world graphs) with thinning, $\mathbf{t}=\mathbf{5}: 5^{\text {th }}$ percentile, median, and $95^{\text {th }}$ percentile of 1,000 estimations are shown for both SRWAVG-DEGREE and RNA-AVG-DEGREE estimators. Estimation results for the sampling fractions ranging from $1 \%$ to $100 \%$ are shown on each plot. Estimation results with three different $\omega$ values are shown on each row: $\omega=[10,50,100]$.

We also conduct average degree estimations with thinning parameter $t=$ 5 for the RNA-AVG-DEGREE and keep $t=1$ for SRW-AVG-DEGREE 
to see whether decreasing the dependency of samples at the expense of the length of the walk improves estimation performance (see Fig 10). Recall that shifted-thinning with the walk size $n$ and the thinning parameter $t$ makes $t$ estimations based on a sample size of $n / t$. Experimental results show that the confidence intervals for RNA-AVG-DEGREE estimator, especially for small sample sizes, are wider. This means that applying the dependency reduction decreases the performance of the RNA-AVG-DEGREE.

Synthetic graphs: To better illustrate the effects of the true average degree, degree variance, and skewness on the estimation performance, we conduct experiments on synthetic graphs with different average degree, degree variance, and skewness. Estimation results and the degree statistics of the synthetic graphs are shown in Fig. 11 and Fig. 12, respectively. The graphs have approximately zero degree assortativity coefficient $(|r|<0.04$ for all synthetic graphs).

The estimation results are shown in Fig 11. We observe that when $\omega=10$ and $\mu_{d}=100$, estimations between SRW-AVG-DEGREE and RNA-AVGDEGREE estimators differ widely in both ER and BA graphs. This result shows that too small $\omega$ in RNA-AVG-DEGREE is not good enough to estimate the reciprocals of the vertex degrees. Recall that the difference between RNA-AVG-DEGREE and SRW-AVG-DEGREE estimators is that RNA-AVG-DEGREE estimator estimates the reciprocals of the vertex degrees while SRW-AVG-DEGREE estimator uses the actual vertex degrees. We also observe that the good selection of $\omega$ is related to the population average degree. For ER graphs with $\mu_{d}=4$ and $\mu_{d}=10$, the best estimation is obtained when $\omega=10$ and increasing $\omega$ decreases the precision for all sampling fractions. However, for the ER graph with $\mu_{d}=100$, the best estimation is obtained when $\omega=100$ and decreasing $\omega$ decreases the precision for all sampling fractions. For the BA graphs with $\mu_{d}=4$ and $\mu_{d}=10$, we still observe that $\omega=10$ is the best option among all three options. However, for the BA graph with $\mu_{d}=100$, the estimation results are almost equal for $f>0.1$. For $f<0.1$, the best estimation is obtained when $\omega=50$ as the walk length is short and approximately $50 \%$ of the nodes have degrees between 50 and 70 (see Fig 12, $B A\left(\mu_{d}=100\right)$ ). This result shows that the optimal selection of $\omega$ not only depends on the average degree but also depends on the degree structure of the underlying graph.

We also conduct experiments on synthetic graphs to see the effect of $\omega$ on the NRMSE of the RNA-AVG-DEGREE estimator (see Fig. 13). We choose $\omega$ in the range $[2,30]$ and $f$ in the range $[0.01,3]$. We perform 1,000 


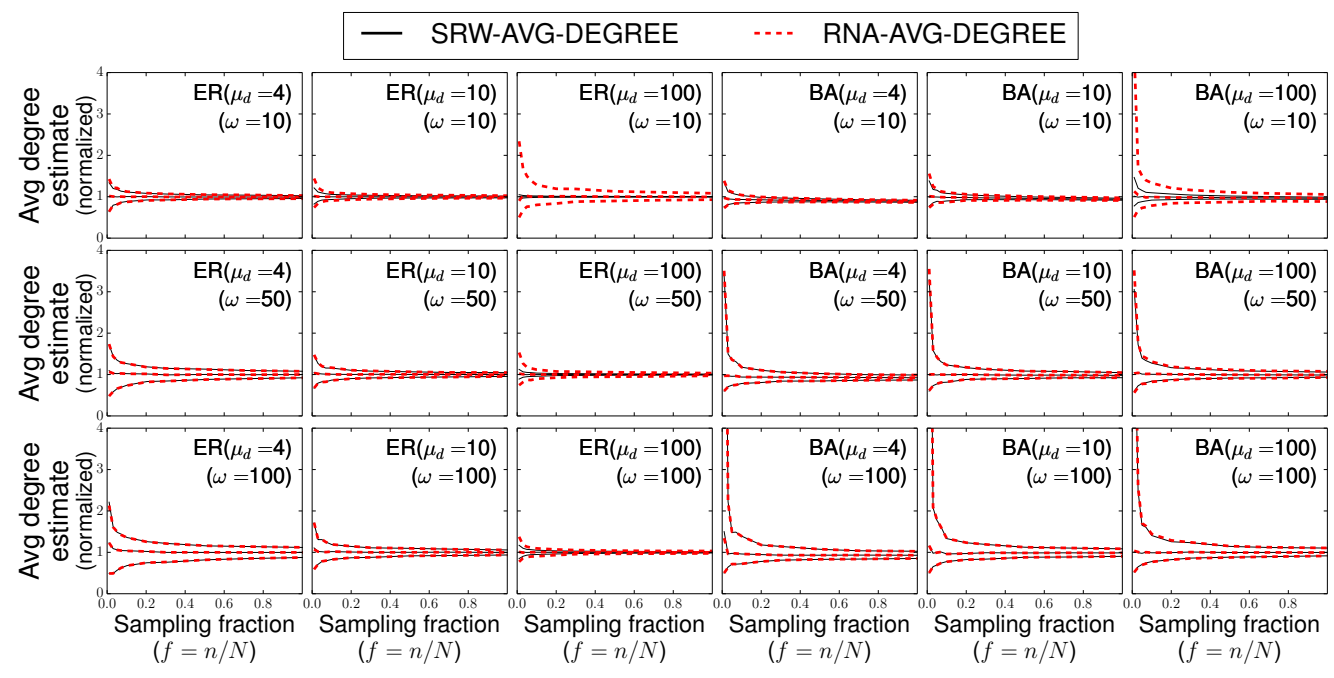

Figure 11: Average degree estimation (synthetic graphs) with no thinning, thinning $=1$ : $5^{t h}$ percentile, median, and $95^{t h}$ percentile of 1,000 estimations are shown for both SRW-AVG-DEGREE and RNA-AVG-DEGREE estimators. Estimation results for the sampling fractions ranging from $1 \%$ to $100 \%$ are shown on each plot. Estimation results with three different $\omega$ values are shown on each row: $\omega=[10,50,100]$.

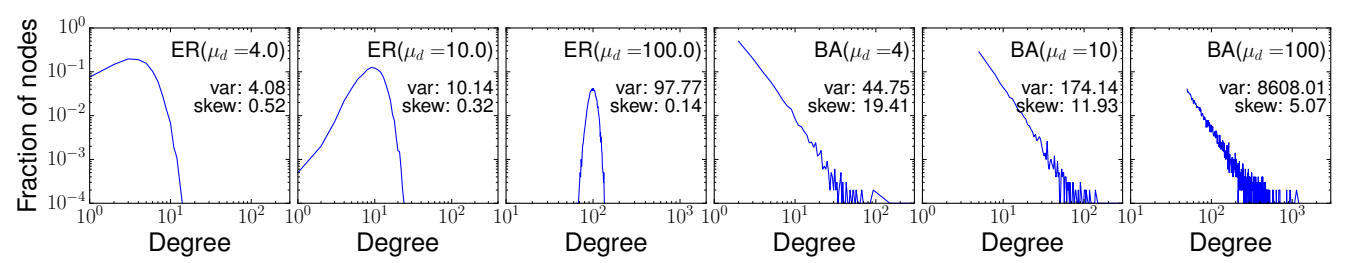

Figure 12: Degree distributions and degree statistics of the synthetic graphs generated by Erdős-Renyi (ER) and Barabási-Albert (BA) models. The variance and the skewness of the degree distribution are abbreviated as var and skew, respectively.

simulations for each sampling fraction and $\omega$. Then, we plot the NRMSE of the estimator. We also plot the average of the best $\omega$ values over 1,000 simulations for each sampling fraction. We see that optimal $\omega$ values highly depend on the degree structure of the underlying graph. We also observe that the optimal value of $\omega$ increases much faster in BA graphs which have higher degree variance and skewness. In ER graph, the optimal value of the $\omega$ is close to the true average degree and increases slightly for the large sample sizes.

A guide on the selection of $\omega$ : The experimental results show that the 


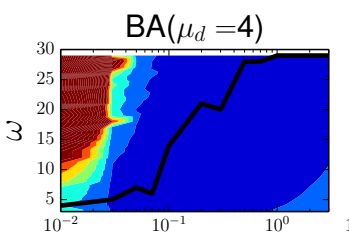

Sampling fraction $(f=n / N)$

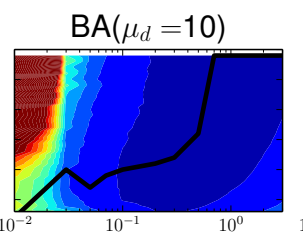

Sampling fraction $(f=n / N)$

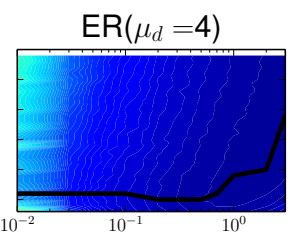

Sampling fraction $(f=n / N)$

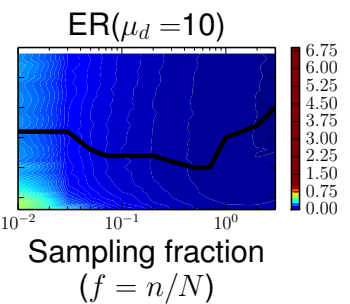

$(f=n / N)$

Figure 13: NRMSE of RNA-AVG-DEGREE for various sampling fractions $0.01<f<3$ and $\omega=[2,30]$. The average of 1,000 independent simulations are shown. The black lines show the average of the best $\omega$ values over 1,000 simulations for each sampling fraction. The best $\omega$ is the one that gives the minimum NRMSE among all $\omega$ values that we use for a given sampling fraction.

selection of $\omega$ has big impact on the estimation performance. We provide a rough guidance on how to select $\omega$. The first important factor in the selection of $\omega$ is the true average degree of the underlying population network. If the underlying population network has a low average degree, $\omega$ should not be too large. Otherwise, we may spend too much sampling budget to estimate individual vertex degrees but we may not visit enough nodes via random walk. For instance, we need to almost double $\omega$ to reduce the relative error of the degree estimation from 0.2 to 0.1 at the $90 \%$ confidence level (see Fig. 5-a). The degree variance of the underlying graph is also an important factor in the selection of $\omega$. To clarify, suppose that the underlying graph is known to have a degree distribution close to uniform distribution. In this case, spending more RN-QUERY calls on estimating individual vertex degrees rather than visiting more nodes via RW would be a good decision. On the other hand, suppose that the underlying graph has a degree distribution with high variance. In this case, estimating individual vertex degrees accurately is not enough to make accurate estimations, the walk should also visit more nodes to explore the underlying graph.

\subsection{Network Size Estimation}

In this section, we evaluate the performance of the network size estimators. We compare the proposed network size estimators with their corresponding SRW-based estimators. More specifically, we compare SRW-HHBNETSIZE estimator with RNA-HHB-NETSIZE estimator by keeping the random walk length the same (similar to the comparison of SRW-AVGDEGREE and RNA-AVG-DEGREE estimators). We also compare SRW- 
VCB-NETSIZE estimator with RNA-MVCB-NETSIZE estimator by keeping the number of RN-QUERY calls the same for both estimators since there is no $\omega$ in RNA-MVCB-NETSIZE estimator. We apply the safety-margin dependency reduction technique in the estimators. We consider three different margin parameters $\{1,10,100\}$.

The summary of results for the average degree estimation is as follows:

- RNA-HHB-NETSIZE estimator with a good selection of $\omega$ outperforms RNA-MVCB-NETSIZE estimator for $f<10$ in real-world graphs.

- RNA-MVCB-NETSIZE estimator is very precise but requires high sampling fraction $(f>10)$ for accurate estimations. Interestingly, for the synthetic graphs, $f=2$ seems large enough for both accurate and precise estimations.

- Applying the safety-margin dependency technique generally improves the accuracy although the precision decreases due to ignoring some sample data, especially for $f<10$.

Real-world Networks: Performance of the SRW-HHB-NETSIZE and RNAHHB-NETSIZE estimators for real-world graphs are shown in Fig. 14. We select $\omega=10$ for RNA-HHB-NETSIZE estimator. Similar to the average degree estimations, the estimators perform almost identical shown as overlapped $90 \%$ confidence intervals in Fig. 14. We observe that confidence intervals get wider as the margin parameter increases. This is expected as the estimators ignore more sample data as the margin parameter increases and the precision decreases.

For both estimators, we observe that increasing margin causes more accurate estimations with less precision especially for $f<10$. For instance, the network size is underestimated with high precision for margin $=1$ for all real-world graphs. However, the estimations are centered around the true network size for margin $=100$ with wider confidence intervals. This illustrates the tradeoff between reducing the dependency and utilizing more data (the bias-variance tradeoff). If we ignore too much data, the precision becomes low but samples become more independent. On the other hand, if we do not ignore enough data, the precision becomes high, but sample becomes more dependent.

Performance of the SRW-VCB-NETSIZE and RNA-MVCB-NETSIZE estimators for real-world graphs are shown in Fig. 15. For RNA-MVCBNETSIZE estimator, we observe very precise but inaccurate estimations for 


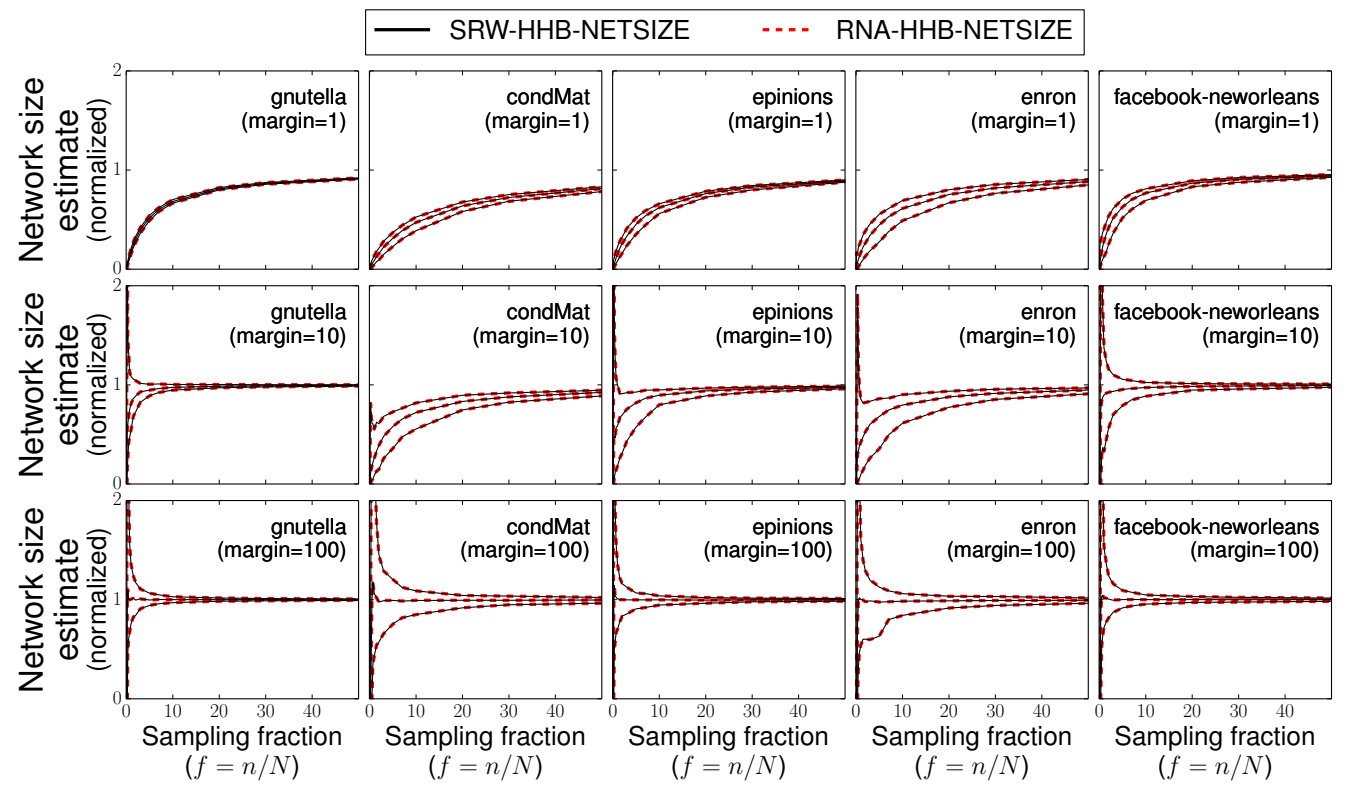

Figure 14: Network size estimation (real-world graphs): $5^{\text {th }}$ percentile, median, and $95^{\text {th }}$ percentile of 1,000 estimations are shown for both SRW-HHB-NETSIZE and RNA-HHBNETSIZE estimators with $t=1$. For RNA-HHB-NETSIZE estimator, $\omega=10$ is used. Estimation results for the sampling fractions ranging from $1 \%$ to $5000 \%$ are shown on each plot. A safety-margin dependency reduction technique with different margin values are shown on each row: margin $=[1,10,100]$.

all graphs that we consider regardless of the margin parameter for $f<10$. The main reason of this observation is that multiplicity values of vertices do not become proportional to the vertex degrees until we have a large enough sample size (see Theorem 4). In SRW-VCB-NETSIZE estimator, we observe the bias-variance tradeoff for small sample sizes. When margin $=1$ (the top row in Fig. 15), we observe an underestimation with a high precision for small sample sizes $(f<0.1)$ for all graphs. When margin $=10$ (the second row), we observe that estimations are not very precise but are around the true parameter. When margin $=100$ (the bottom row), we observe much less precision due to ignoring too much data. In these graphs, margin $=10$ seems to be better than the others. We conjecture that this is directly related to the average degree of the considered real-world graphs being close to 10 . Synthetic graphs: Experimental results for SRW-HHB-NETSIZE and RNAHHB-NETSIZE estimators on synthetic graphs are shown in Fig. 16. We 


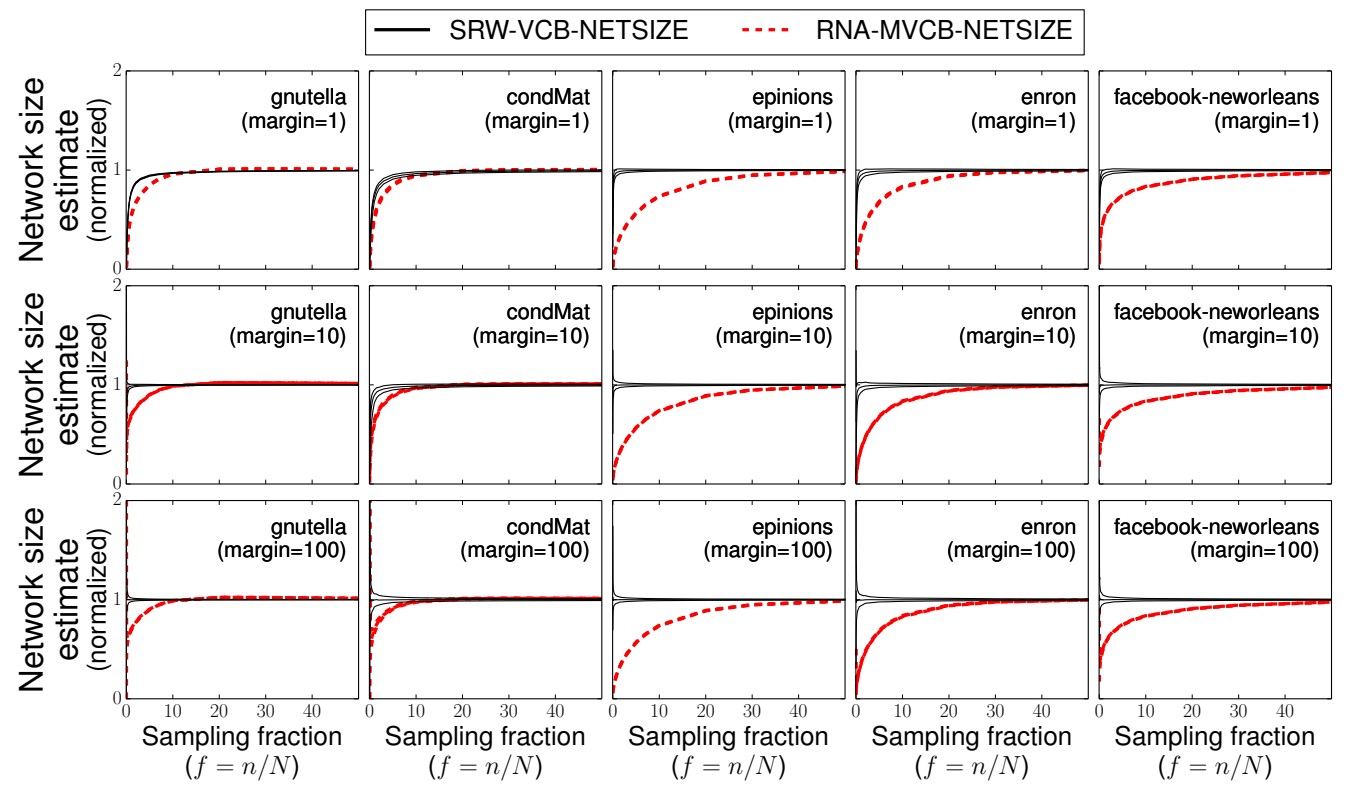

Figure 15: Network size estimation (real-world graphs): $5^{\text {th }}$ percentile, median, and $95^{\text {th }}$ percentile of 1,000 estimations are shown for both SRW-VCB-NETSIZE and RNA-MVCBNETSIZE estimators. Estimation results for the sampling fractions ranging from $1 \%$ to $5000 \%$ are shown on each plot. A safety-margin dependency reduction technique with different margin values are shown on each row: margin $=[1,10,100]$.

observe that the estimation performance does not differ between ER and BA graphs when the average degree and margin parameters are the same although the graphs are structurally different. For margin $=1$ case, we see underestimation when $\mu_{d}=4$ and $\mu_{d}=10$ for $f<20$ in both ER and BA graphs. This can be explained by the underestimation of $M$ due to small $n_{\omega}$ and large number of collisions due to small average degree in Eq. 11. We do not observe underestimation when $f>1$ and $\mu_{d}=100$. This might be due to the balance of the underestimation of $M$ and small $C_{E}$ due to high average degree. When we increase margin to 10 or 100 , we see that estimations for $\mu_{d}=4$ or $\mu_{d}=10$ cases in both ER and BA graphs are more accurate and precise than that of $\mu_{d}=100$. These results illustrate that optimal margin parameter depends on the average degree of the underlying population graph as well as the sampling fraction.

Experimental results for SRW-VCB-NETSIZE and RNA-MVCB-NETSIZE estimators on synthetic graphs are shown in Fig. 17. Similar to the estima- 


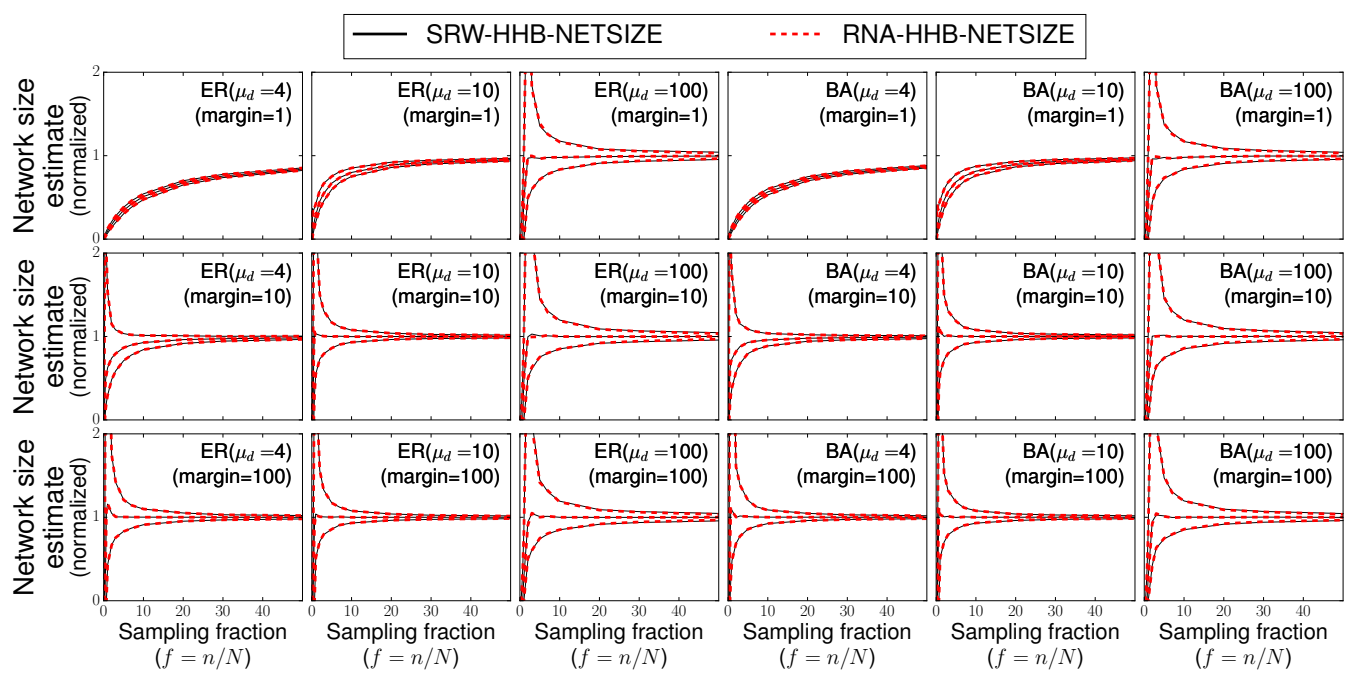

Figure 16: Network size estimation (synthetic graphs): $5^{\text {th }}$ percentile, median, and $95^{\text {th }}$ percentile of 1,000 estimations are shown for both SRW-HHB-NETSIZE and RNA-HHBNETSIZE estimators with $t=1$. For RNA-HHB-NETSIZE estimator, $\omega=10$ is used. Estimation results for the sampling fractions ranging from $1 \%$ to $5000 \%$ are shown on each plot. A safety-margin dependency reduction technique with different margin values are shown on each row: margin $=[1,10,100]$.

tion results on real-world graphs, all estimations on synthetic graphs are very precise especially when sampling fraction $f>1$. A very interesting observation is that RNA-MVCB-NETSIZE estimator is very accurate and precise when $f>1$ and outperforms SRW-VCB-NETSIZE estimator. We also observe that SRW-VCB-NETSIZE estimator tends to overestimate the network size when $1<f<30$ on ER graphs, while it underestimates the network size when $0.1<f<5$ on BA graphs. Therefore, when $<1<f<5$, we consistently underestimate the network size in ER graphs, while we consistently overestimate the network size. This creates a practical challenge as the degree structure of the graph may cause consistent underestimations or overestimations. Without knowing the degree structure, we may not decide to which case the estimations belong to. Recall that in RNA-HHB-NETSIZE and SRW-HHB-NETSIZE estimators, degree structure did not affect the estimation accuracy as much. The selection of margin parameter and the average degree of the underlying graph was the main factors affecting the performance of the estimators.

Comparison of RNA-HHB-NETSIZE with RNA-MVCB-NETSIZE: 


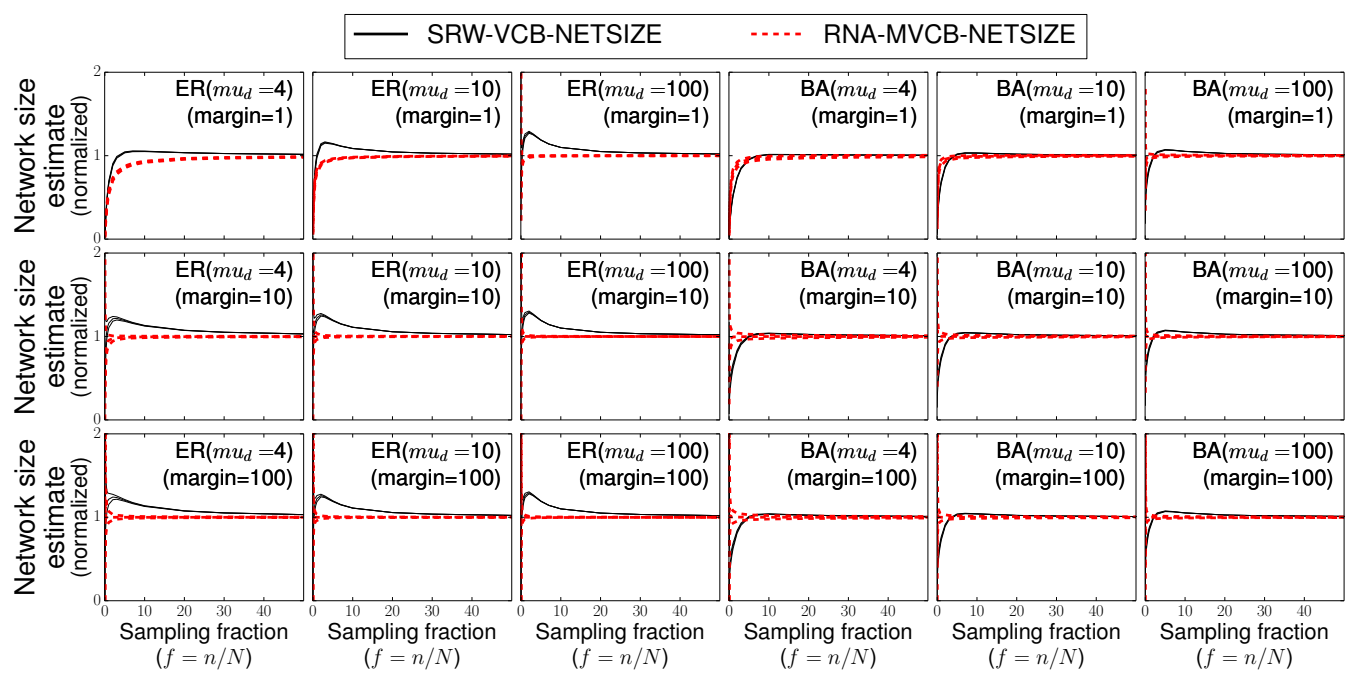

Figure 17: Network size estimation (synthetic graphs): $5^{\text {th }}$ percentile, median, and $95^{\text {th }}$ percentile of 1,000 estimations are shown for both SRW-VCB-NETSIZE and RNA-MVCBNETSIZE estimators. Estimation results for the sampling fractions ranging from $1 \%$ to $5000 \%$ are shown on each plot. A safety-margin dependency reduction technique with different margin values are shown on each row: margin $=[1,10,100]$.

In real-world graphs, we observe that with a good selection of $\omega$ (e.g., $\omega=$ 100), RNA-HHB-NETSIZE outperforms RNA-MVCB-NETSIZE in terms of accuracy when $f<10$ (see Fig. 14 and Fig. 15 ). However, when $f \approx 10$, we see that RNA-MVCB-NETSIZE estimator starts making estimations with high precision and accuracy (see GNUTELLA and CONDMAT in Fig. 15). In the synthetic graphs, we observe that RNA-HHB-NETSIZE estimator is very precise and accurate when $f>20$ (even larger in some graphs, e.g., $E R\left(\mu_{d}=100\right)$ and $B A\left(\mu_{d}=100\right)$ in Fig. 16), while the similar performance is obtained in RNA-MVCB-NETSIZE when $f>2$ and margin $>10$ (see Fig. 17).

\section{Discussion}

In this section, we discuss several practical issues related to our problem context including adaptive number of RN-QUERY calls per vertex and estimation in dynamic graphs. 


\subsection{General Discussion on Practical Issues}

One of the assumptions of our estimators is that the sample is collected by a stationary random walk. In practice, this can be achieved by ignoring a number of initial steps in the random walk (known as burn-in period). In general, the number of steps until convergence, i.e., the mixing time, depends on the underlying structure of the graph and can be bounded by a function of the modulus of the second largest eigenvalue of the graph. Mohaisen et al. [78] study this dependency and the mixing time of various OSN graphs. Gjoka et al. [8] apply two formal convergence diagnostic techniques on OSN graphs. Note that the burn-in period is relevant for both RSRW and ERSRW sampling schemes as they are just variations of a random walk.

To estimate the network size, one can be tempted to save unique vertex ids visited by SRW until no new ids are obtained instead of using the multiplicity based estimator which requires high sample sizes $(f \approx 10)$. However, this idea has much higher cost $((1-o(1)) \log n<f<O(4 m))$ for general undirected and non-bipartite graphs ${ }^{10}[28]$; therefore, we do not consider a complete topology discovery as a viable alternative.

Another point is that any nodal property that is not correlated with the vertex ids are impossible to estimate under the RNA model. For instance, if we study the average age of an OSN users, we should either observe or estimate the age of each sampled user together with its degree in order to correct the bias. Even though we can estimate the vertex degrees under the RNA model, estimating the age of a user (and hence the bias correction) is not possible as there is no information revealed by the RN-QUERY.

\subsection{Estimation with Adaptive Number of RN-QUERY Calls}

Note that our proposed estimators use a sampling scheme that makes a fixed number of RN-QUERY calls per visited vertex. In this section, we propose average degree and network size estimators that use an alternative sampling scheme making variable number of RN-QUERY calls per visited vertex. Finally, we run simulation experiments using our real-world graphs to evaluate the performance of these estimators as compared to the estimators using fixed number of RN-QUERY calls.

\footnotetext{
${ }^{10}$ Time to visit all vertices in a graph with a SRW is also known as the cover time of the graph.
} 


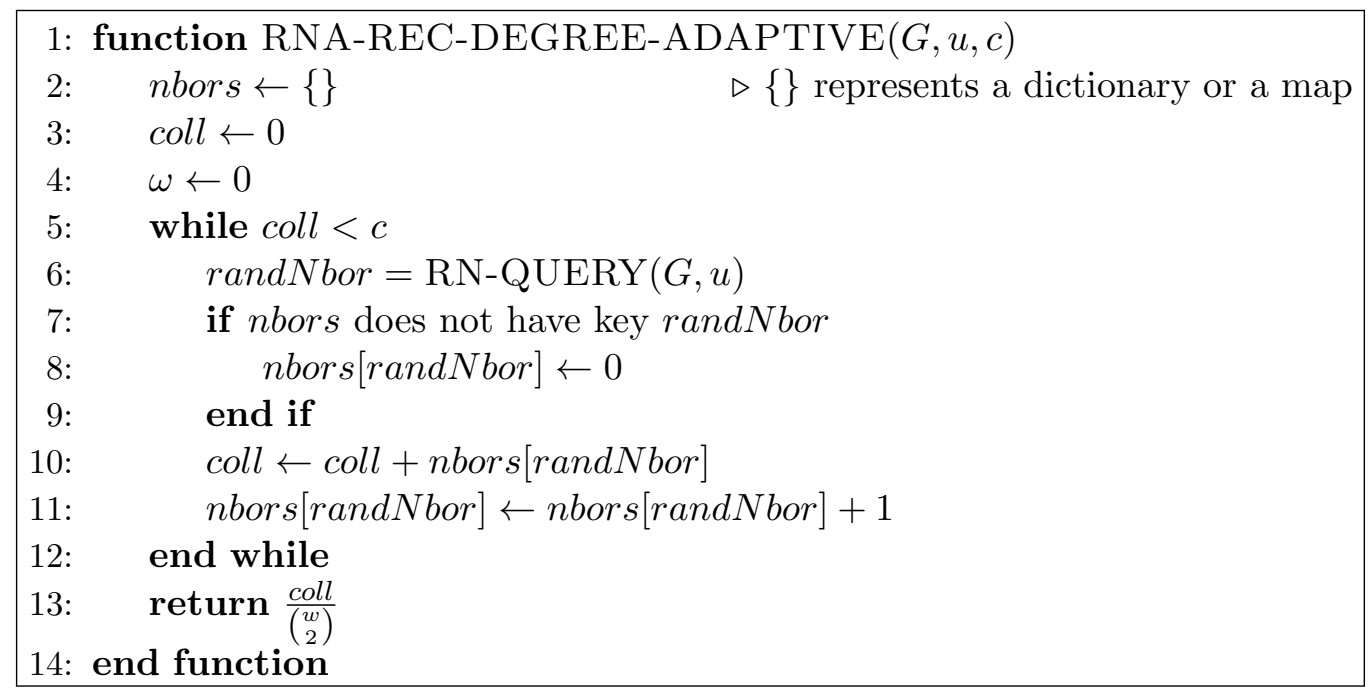

Algorithm 6: On-the-fly estimator for the reciprocal of the degree of the vertex $u$ under ERSRW sampling with adaptive probing.

ERSRW with Adaptive Probing: In this sampling design, RN-QUERY calls are made for the current vertex in the walk until the number of collisions exceeds the threshold $c$. Note that this type of probing is more likely to make more RN-QUERY calls for a vertex with high degree. However, it is not obvious whether this property improves the overall performance of the estimation. The walk is biased towards high degree nodes and these nodes are more likely to consume more sampling budget. Therefore, the walker may visit less number of vertices. Basically, there is a tradeoff between visiting many nodes and better reciprocal degree estimation for high degree vertices.

Algorithm 6 shows the pseudocode of the estimator for the reciprocals of the vertex degrees. Note that the estimator is very similar to RNA-RECDEGREE but this time $\omega$ is not fixed. In our simulations, we use RNAREC-DEGREE-ADAPTIVE, instead of RNA-REC-DEGREE, as a subroutine in the average degree and the network size estimations. We call the corresponding estimators as RNA-AVG-DEGREE-ADAPTIVE and RNAHHB-NETSIZE-ADAPTIVE, respectively.

Fig. 18 and Fig. 19 show our simulation results. The main findings are as follows: estimation accuracy is generally worse compared to the RNAAVG-DEGREE and RNA-HHB-NETSIZE estimators that use ERSRW with fixed probing. The parameter $c$ has a crucial effect on the bias-variance 


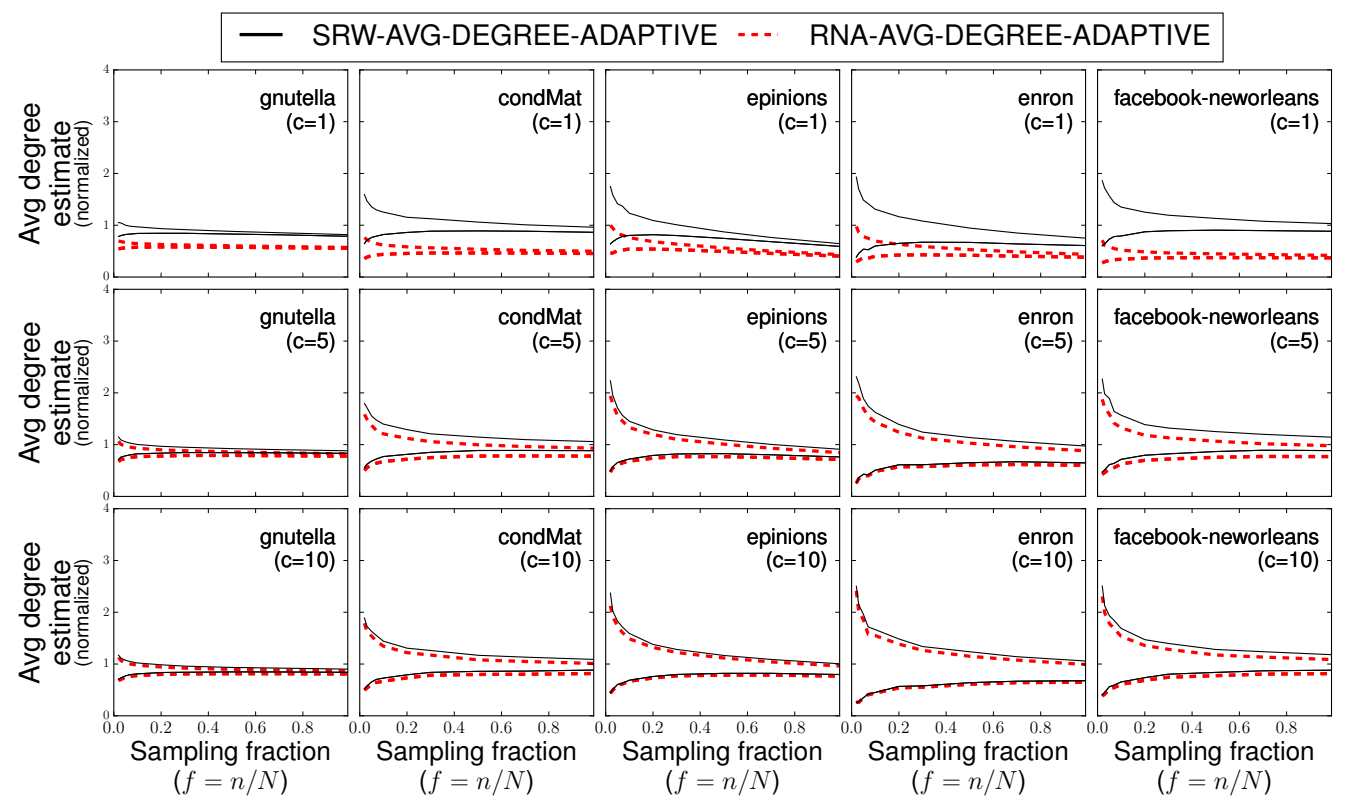

Figure 18: Average degree estimation (synthetic graphs) with no thinning, thinning $=1$ : $5^{\text {th }}$ percentile, median, and $95^{\text {th }}$ percentile of 1,000 estimations are shown.

tradeoff. Choosing too small $c$ gives an underestimation of the average degree with high precision while choosing large $c$ gives a less biased estimation with higher variance (see Fig. 18). We also observe the same phenomena in the estimation of the network size (see Fig. 19).

\subsection{Estimation on Dynamic Graphs}

One of the assumptions of our estimators is that the underlying network is static. However, it is still important to understand when our statistical model break and estimations become inaccurate. We perform a simulation study on a publicly available real-world social graph data which represents the friendship relation of the users of a social news website named digg.com [79]. The dataset contains a total of 1.7 million friendship edges and their creation time between August 2005 and July 2009.

We perform the experiment as follows: 1) For the first 10 days, we initialize the network without sampling. 2) Then, we make RN-QUERY calls periodically, which is controlled by a parameter named query interval. 3) We estimate the average degree every 24 hours using RNA-AVG-DEGREEADAPTIVE estimator with $c=10$. While performing the average degree esti- 


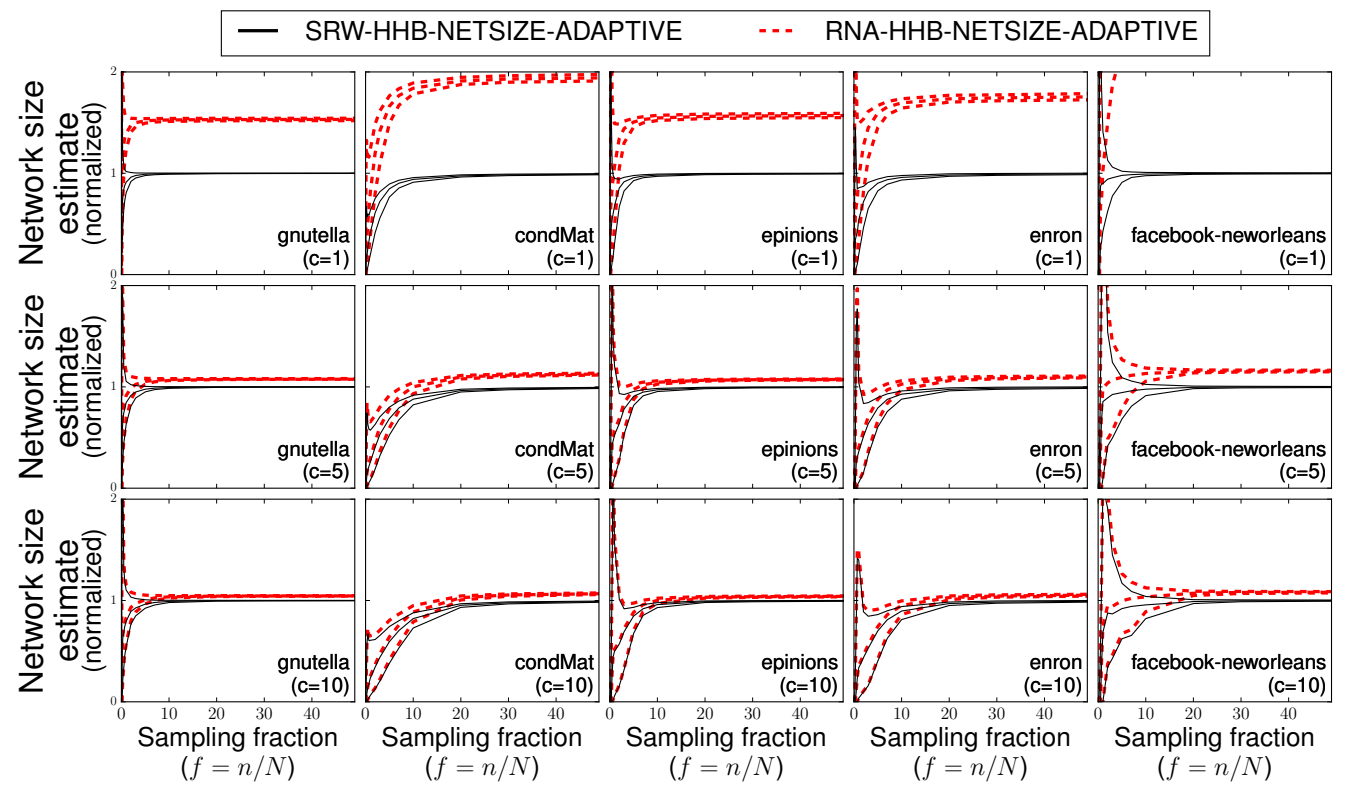

Figure 19: Network size estimation (real-world graphs): $5^{\text {th }}$ percentile, median, and $95^{\text {th }}$ percentile of 1,000 estimations are shown for both SRW-HHB-NETSIZE-ADAPTIVE and RNA-HHB-NETSIZE-ADAPTIVE estimators with $t=1$. For RNA-HHB-NETSIZEADAPTIVE estimator, $c=10$ and margin $=10$ is used.

mation, we only use the degree estimations of the last couple of nodes, which is controlled by the window size parameter. Fig. 20 illustrates the estimation results where $\mathrm{x}$-axis represents the simulation time since the beginning of the experiment. We see that the average degree of the graph does not change much in the first 800 days and then it gradually increases. When the true average degree does not change over time, we observe that estimations perform almost the same and the effects of the parameters are not observable. In the rest, we focus on the days after the true average degree starts increasing.

First, we observe the effect of window size on the bias-variance tradeoff. When the window size is too small, we observe high variance in the estimation, which can be attributed to the small sample size considered in the estimation. When the window size is too large, we observe a bias in the estimation as the past data is not representative of the current network state. Note that it is intuitive that when the actual average degree increases (decreases) over time, using a large window size causes underestimation (overestimation). 


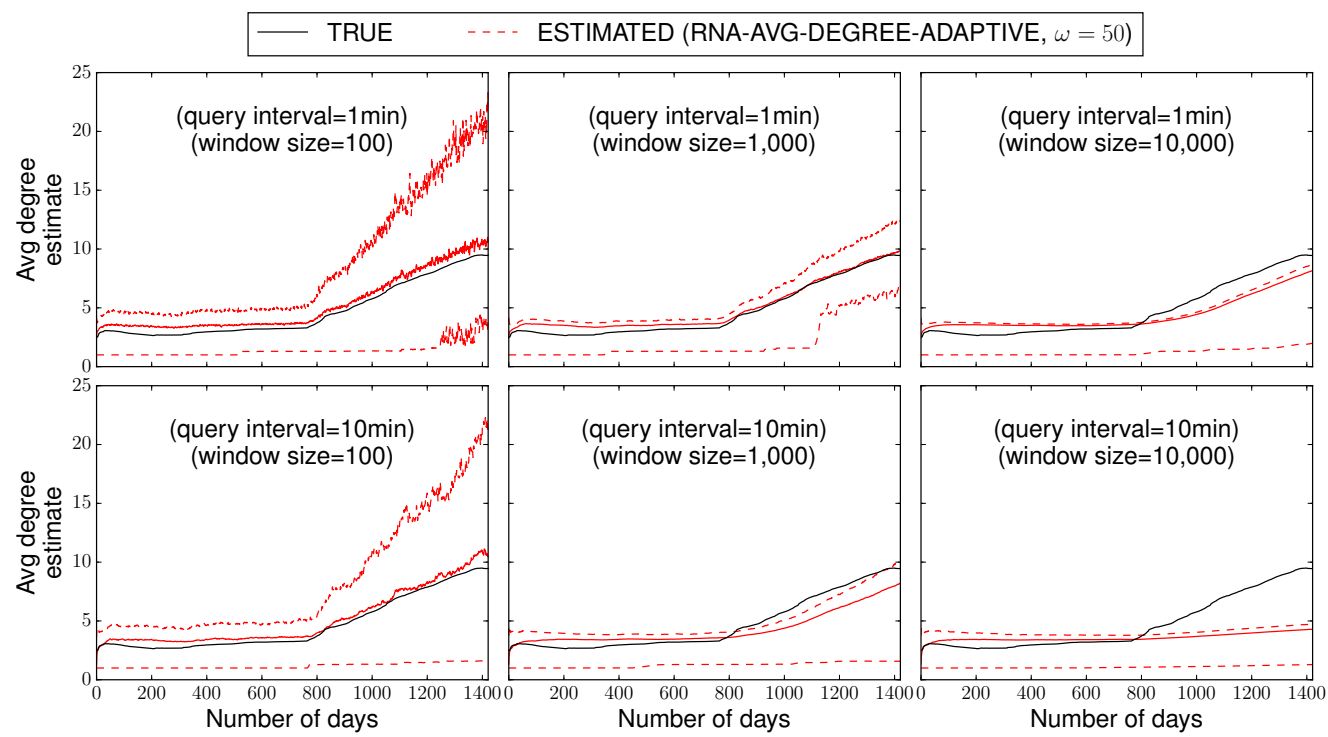

Figure 20: Average degree estimation of dynamic friendship graph of digg.com, a social news website, over the period of approximately 4 years (from August 2005 to July 2009). Red dotted lines represent the 95th percentile, median, and the 5th percentile of 1000 estimations per day from top to bottom in each graph. The graph illustrates the effects of the estimation parameters on the bias-variance tradeoff over time. We use the abbreviation min for minutes in the query interval.

The selection of query interval is also important as it determines (together with the window size parameter) how representative our sample data used in the estimation. Basically, low query interval value helps to take samples fast and the window fills fast with more recent data; and hence the sample is more representative of the current data. We observe that the increase in the estimation is not enough to catch the true value when the query interval is 10 minutes and window size is 1,000 or 10,000. When the query interval is reduced to 1 minute, we observe that the increase in the estimation is better in catching the true value although with the window size $=10,000$ still results in an underestimation.

In summary, the estimation accuracy highly depends on how fast the true value changes and whether the change has certain patterns such as gradual increase or decrease. In addition, the limitations imposed by the OSN service providers on the frequency of queries affects the estimation accuracy. Note that these factors are independent of the sampling design. In order to achieve a good accuracy, the sampling design parameters such as query interval and 
window size should be selected carefully.

\section{Conclusion}

In this paper, we have proposed sampling schemes and estimators for the network size and the average degree properties under the RNA model. Using simulations on both real-world and synthetic graphs, we have demonstrated that network size estimation requires high sampling fractions $(f>10)$ under the RNA model for accurate and precise estimations. However, the average degree can be estimated with high accuracy and precision using practical sample sizes $(f<0.2)$ with a reasonable selection of sampling design parameter. We have also empirically shown that applying dependency reduction technique on the average degree estimator decreases the precision; while applying it in the network size estimators may increase the accuracy of the estimations. We also discussed the several practical issues of estimation under the RNA model. We believe this study will motivate future studies to develop estimators under various other interesting sampling designs and not to stick with the typical sampling designs such as independent vertex/edge sampling or induced subgraph sampling when they are not efficient under the specific problem constraints.

\section{Appendix A. The Ergodic Theorem}

Theorem 4 ([34], Theorem 1.10.2). Let $C$ be a finite, irreducible, aperiodic Markov Chain and $\Delta(i, t)$ be the number of visits to vertex $i$ in first $t$ steps. Let $\tau_{d}=\sum_{i=1}^{N} d_{i}$ be the sum of the vertex degrees. Then,

$$
\Delta(i, t) \stackrel{\text { a.s. }}{\longrightarrow} t d_{i} / \tau_{d}
$$

where $\stackrel{\text { a.s. }}{\longrightarrow}$ denotes almost sure convergence.

The main implication of the Ergodic Theorem is that the multiplicity of a vertex becomes proportional to its degree in the limit of a large sample sizes. Although this is an asymptotic result, i.e., it holds as the sample size goes to infinity, it is an important property to make use of under the very limited data access model that we consider. Note that RNA-MVCB-NETSIZE estimator needs a property that is proportional to the vertex degree and we choose this property as the vertex multiplicity based on the Ergodic Theorem. 


\section{Acknowledgement}

This research was supported by the National Science Foundation under Award No. 1027520.

\section{References}

[1] A. Dasgupta, R. Kumar, T. Sarlos, On Estimating the Average Degree, in: 23nd International World Wide Web Conference (WWW), Seoul, Korea, 2014, pp. 795-806.

[2] L. Katzir, E. Liberty, O. Somekh, Estimating Sizes of Social Networks via Biased Sampling, in: Proceedings of the 20th International Conference on World Wide Web (WWW), 2011, pp. 597-606.

[3] S. J. Hardiman, L. Katzir, Estimating clustering coefficients and size of social networks via random walk, in: Proceedings of the 22nd International Conference on World Wide Web (WWW), 2013, pp. 539-549.

[4] S. Ye, F. Wu, Estimating the Size of Online Social Networks, in: SocialCom, 2010, pp. 169-176.

[5] M. Kurant, M. Gjoka, Y. Wang, Z. W. Almquist, C. T. Butts, A. Markopoulou, Coarse-grained topology estimation via graph sampling, in: Proceedings of the 5th ACM workshop on Online Social Networks (WOSN), 2012, pp. 25-30.

[6] M. Kurant, C. T. Butts, A. Markopoulou, Graph Size Estimation.

[7] M. Kurant, M. Gjoka, C. T. Butts, A. Markopoulou, Walking on a Graph with a Magnifying Glass: Stratified Sampling via Weighted Random Walks, in: Proceedings of the ACM SIGMETRICS Joint International Conference on Measurement and Modeling of Computer Systems, Vol. 1, 2011, pp. 281-292.

[8] M. Gjoka, M. Kurant, C. T. Butts, A. Markopoulou, Practical Recommendations on Crawling Online Social Networks, IEEE Journal On Selected Areas in Communications 29 (9) (2011) 1872-1892. 
[9] M. Gjoka, C. T. Butts, M. Kurant, A. Markopoulou, Multigraph sampling of online social networks, IEEE Journal on Selected Areas in Communications 29 (2011) 1893-1905.

[10] M. Sirivianos, K. Kim, X. Yang, SocialFilter: Introducing social trust to collaborative spam mitigation, in: Proceedings of the 2010 international conference on Collaborative methods for security and privacy (CollSec), Shanghai, China, 2015.

[11] A. Mislove, A. Post, P. Druschel, K. P. Gummadi, Ostra: Leveraging Trust to Thwart Unwanted Communication, in: NSDI, San Francisco, CA, 2008.

[12] S. Garriss, M. Kaminsky, M. Freedman, B. Karp, D. Mazieres, H. Yu, Re: Reliable Email, in: NSDI, San Jose, CA, 2006, pp. 22-22.

[13] H. Yu, M. Kaminsky, P. B. Gibbons, A. D. Flaxman, SybilGuard: Defending against sybil attacks via social networks, IEEE/ACM Transactions on Networking 16 (3) (2008) 576-589.

[14] A. Erola, J. Castellà-Roca, A. Viejo, J. M. Mateo-Sanz, Exploiting social networks to provide privacy in personalized web search, Journal of Systems and Software 84 (2011) 1734-1745.

[15] D. Chau, S. Pandit, S. Wang, C. Faloutsos, Parallel crawling for online social networks, in: Proceedings of the 16th International Conference on World Wide Web (WWW), 2007, pp. 1283-1284.

[16] R. Dey, Z. Jelveh, K. Ross, Facebook users have become much more private: A large-scale study, in: IEEE International Conference on Pervasive Computing and Communications Workshops, (PERCOM), Lugano, Switzerland, 2012, pp. 346-352.

[17] L. Massoulié, E. Le Merrer, A.-M. Kermarrec, A. Ganesh, Peer counting and sampling in overlay networks: Random Walk Methods, in: Proceedings of the twenty-fifth annual ACM symposium on Principles of distributed computing (PODC), 2006, pp. 123-132.

[18] K. Avrachenkov, B. Ribeiro, D. Towsley, Improving Random Walk Estimation Accuracy with Uniform Restarts, in: Proceedings of the 17th 
Workshop on Algorithms and Models for the Web Graph, 2010, pp. 98-109.

[19] B. Ribeiro, D. Towsley, Estimating and Sampling Graphs with Multidimensional Random Walks, in: Proceedings of the 10th ACM SIGCOMM Conference on Internet Measurement (IMC), 2010, pp. 390-403.

[20] B. Ribeiro, P. Wang, F. Murai, D. Towsley, Sampling Directed Graphs with Random Walks, in: Proceedings of the 31st conference on Information communications (INFOCOM), 2012, pp. 1692-1700.

[21] M. Gjoka, M. Kurant, C. T. Butts, A. Markopoulou, Walking in facebook: A case study of unbiased sampling of OSNs, in: Proceedings of the 29th conference on Information communications (INFOCOM), 2010, pp. 1-9.

[22] X. Xu, C.-H. Lee, D. Y. Eun, A general framework of hybrid graph sampling for complex network analysis, in: Proceedings of the 33rd conference on Information communications (INFOCOM), 2014, pp. 27952803.

[23] P. Wang, J. Zhao, J. C. S. Lui, D. Towsley, X. Guan, Sampling node pairs over large graphs, in: IEEE 29th International Conference on Data Engineering (ICDE), no. 1, 2013, pp. 781-792.

[24] D. D. Heckathorn, Respondent-driven sampling: A new approach to the study of hidden populations, Social Problems 44 (2) (1997) 174-199.

[25] M. J. Salganik, D. D. Heckathorn, Sampling and estimation in hidden populations using respondent-driven sampling, Sociological methodology 34 (2004) (2004) 193-240.

[26] M. Kurant, A. Markopoulou, P. Thiran, Towards unbiased BFS sampling, IEEE Journal on Selected Areas in Communications 29 (9) (2011) 1799-1809.

[27] M. Kurant, A. Markopoulou, P. Thiran, On the bias of BFS (Breadth First Search), in: 22nd International Teletraffic Congress (1TC 22), IEEE, Amsterdam, Netherlands, 2010, pp. 1-8. 
[28] L. Lovász, Random walks on graphs: A survey, Combinatorics, Paul erdos is eighty 2 (1) (1993) 1-46.

[29] M. M. Zhang, N. N. Zhang, G. G. Das, Mining a search engine's corpus without a query pool, in: International Conference on Information and Knowledge Management, 2013, pp. 29-38.

[30] D. Stutzbach, R. Rejaie, N. Duffield, S. Sen, W. Willinger, Sampling Techniques for Large, Dynamic Graphs, in: Proceedings of the 25th conference on Information communications (INFOCOM), 2006, pp. 16.

[31] A. H. Rasti, M. Torkjazi, R. Rejaie, N. Duffield, W. Willinger, D. Stutzbach, Respondent-driven sampling for characterizing unstructured overlays, in: Proceedings of the 28th conference on Information communications (INFOCOM), 2009, pp. 2701-2705.

[32] M. R. Henzinger, A. Heydon, M. Mitzenmacher, M. Najork, On nearuniform URL sampling, Computer Networks 33 (1-6) (2000) 295-308.

[33] J. Leskovec, C. Faloutsos, Sampling from large graphs, in: Proceedings of the 12th ACM SIGKDD international conference on Knowledge discovery and data mining, 2006, pp. 631-636.

[34] J. R. Norris, Markov Chains, no. 2008 in Cambridge series in statistical and probabilistic mathematics, Cambridge University Press, 1999.

[35] S. K. Thompson, Sampling, 3rd Edition, Wiley Series in Probability and Statistics, John Wiley \& Sons, Inc., Hoboken, NJ, USA, 2012.

[36] J. Lu, H. Wang, Variance reduction in large graph sampling, Information Processing and Management 50 (3) (2014) 476-491.

[37] D. Rasch, The estimation of animal abundance and related parameters, Blackburn Press, 1982.

[38] J. Lu, D. Li, Bias Correction in a Small Sample from Big Data, TKDE 25 (11) (2012) 2658-2663.

[39] C. Doerr, N. Blenn, Metric convergence in social network sampling, in: Proceedings of the 5th ACM workshop on HotPlanet (HotPlanet), 2013, pp. $45-50$. 
[40] S. Ye, J. Lang, F. Wu, Crawling online social graphs, in: APWeb, 2010, pp. 236-242.

[41] C. Wilson, B. Boe, A. Sala, K. P. Puttaswamy, B. Y. Zhao, User interactions in social networks and their implications, in: Proceedings of the fourth ACM european conference on Computer systems (EuroSys), Nuremberg, 2009, pp. 205-218.

[42] A. Mislove, M. Marcon, K. P. Gummadi, P. Druschel, B. Bhattacharjee, Measurement and analysis of online social networks, in: Proceedings of the 7th ACM SIGCOMM Conference on Internet Measurement (IMC), 2007, pp. 29-42.

[43] Y.-Y. Ahn, S. Han, H. Kwak, S. Moon, H. Jeong, Analysis of Topological Characteristics of Huge Online Social Networking Services, in: Proceedings of the 16th International Conference on World Wide Web (WWW), 2007, pp. 835-844.

[44] V. Krishnamurthy, M. Faloutsos, M. Chrobak, L. Lao, Reducing Large Internet Topologies, in: Proceedings of the 4th IFIP-TC6 international conference on Networking Technologies, Services, and Protocols; Performance of Computer and Communication Networks; Mobile and Wireless Communication Systems, Vol. 3462, 2005, pp. 328-341.

[45] S. Wasserman, K. Faust, Social Network Analysis Methods and Applications, 1st Edition, Cambridge University Press, 1994.

[46] A. Leon-Garcia, Probability, statistics, and random processes for electrical engineering, 3rd Edition, 2008.

[47] N. Metropolis, A. W. Rosenbluth, M. N. Rosenbluth, A. H. Teller, E. Teller, Equation of State Calculations by Fast Computing Machines, The Journal of Chemical Physics 21 (1953) 1087-1092. arXiv:5744249209.

[48] O. Frank, Survey Sampling in Graphs, Journal of Statistical Planning and Inference 1 (3) (1977) 235-264.

[49] O. Frank, Sampling and estimation in large social networks, Social networks 22 (1979) 91-101. 
[50] O. Frank, Moment Properties of Subgraph Counts in Stochastic Graphs, Annals of the New York Academy of Sciences 319 (1) (1979) 207-218.

[51] O. Frank, Sampling and Inference in a Population Graph, International Statistical Review 48 (1) (1980) 33-41.

[52] O. Frank, Random sampling and social networks. A survey of various approaches, Mathématiques et Sciences Humaines 26 (104) (1988) 19 33.

[53] M. Granovetter, T. American, N. May, Network Sampling : Some First Steps, American Journal of Sociology 81 (6) (1976) 1287-1303.

[54] M. Capobianco, O. Frank, Comparison of Statistical Graph-size Estimators, Journal of Statistical Planning and Inference 6 (1982) 87-97.

[55] L. A. Goodman, Snowball sampling, The Annals of Mathematical Statistics 32 (1) (1961) 148-170.

[56] M. S. Handcock, K. J. Gile, Modeling social networks from sampled data, The Annals of Applied Statistics 4 (1) (2010) 5-25.

[57] K. J. Gile, M. S. Handcock, Respondent Driven Sampling: An Assessment of Current Methodology, Sociological Methodology 40 (2010) 285327.

[58] K. J. Gile, L. G. Johnston, M. J. Salganik, Diagnostics for respondentdriven sampling, Journal of the Royal Statistical Society: Series A (Statistics in Society) 178 (1) (2015) 241-269.

[59] E. Volz, D. D. Heckathorn, Probability based estimation theory for respondent driven sampling, Journal of Official Statistics, 24 (1) (2008) 79-97.

[60] P. V. Marsden, Network data and measurement, Annual Review of Sociology 16 (1990) 435-463.

[61] M. Newman, Ego-centered networks and the ripple effect, Social Networks 25 (1) (2003) 83-95. 
[62] P. Wang, B. Ribeiro, J. Zhao, J. C. S. Lui, D. Towsley, X. Guan, Practical Characterization of Large Networks Using Neighborhood Information (nov 2013).

[63] S. J. Hardiman, P. Richmond, S. Hutzler, Calculating statistics of complex networks through random walks with an application to the on-line social network Bebo, The European Physical Journal B 71 (4) (2009) 611-622.

[64] K. Bharat, A. Broder, A Technique for Measuring the Relative Size and Overlap of Public Web Search Engines, Computer Networks and ISDN Systems 30 (1-7) (1998) 379-388.

[65] Z. Bar-Yossef, M. Gurevich, Random sampling from a search engine's index, Journal of the ACM (JACM) 55 (5) (2008) 1-74.

[66] M. P. Stumpf, C. Wiuf, Sampling properties of random graphs: The degree distribution, Physical Review E 72 (3) (2005) 036118.

[67] M. P. Stumpf, C. Wiuf, R. May, Subnets of scale-free networks are not scale-free, PNAS 102 (12) (2005) 4221-4224.

[68] S. Lee, P.-J. Kim, H. Jeong, Statistical properties of sampled networks, Physical Review E 73 (1) (2006) 016102.

[69] C.-h. Lee, X. Xu, D. Y. Eun, Beyond random walk and metropolishastings samplers, in: ACM SIGMETRICS Performance Evaluation Review, Vol. 40, 2012, pp. 319-330.

[70] N. K. Ahmed, J. Neville, R. Kompella, Reconsidering the Foundations of Network Sampling, in: Proceedings of Workshop on Information in Networks, 2010.

[71] E. M. Airoldi, K. M. Carley, Sampling algorithms for pure network topologies, ACM SIGKDD Explorations Newsletter 7 (2) (2005) 13-22.

[72] N. Ahmed, J. Neville, R. R. Kompella, Network Sampling via Edgebased Node Selection with Graph Induction, Tech. rep., CSD TR 11-016, Computer Science Department, Purdue University (2011). 
[73] E. Cem, M. E. Tozal, K. Sarac, Impact of sampling design in estimation of graph characteristics, in: IEEE 32nd International Performance Computing and Communications Conference (IPCCC), 2013, pp. 1-10.

[74] D. Stutzbach, R. Rejaie, N. Duffield, S. Sen, W. Willinger, On Unbiased Sampling for Unstructured Peer-to-Peer Networks, IEEE/ACM Transactions on Networking 17 (2) (2009) 377-390.

[75] W. Willinger, R. Rejaie, M. Torkjazi, M. Valafar, M. Maggioni, Research on online social networks, ACM SIGMETRICS Performance Evaluation Review 37 (2) (2010) 49.

[76] J. Leskovec, A. Krevl, SNAP Datasets (jun 2014).

URL http://snap.stanford.edu/data

[77] B. Viswanath, A. Mislove, M. Cha, K. P. Gummadi, On the Evolution of User Interaction in Facebook, in: Proceedings of the 2nd ACM workshop on Online Social Networks (WOSN), 2009, pp. 37-42.

[78] A. Mohaisen, A. Yun, Y. Kim, Measuring the Mixing Time of Social Graphs, in: Science And Technology, Vol. 40, 2010, pp. 383-389.

[79] J. Kunegis, Digg Friendship Graph 2009 (2016).

URL http://konect. uni-koblenz.de/networks/digg-friends 


\section{The Biography of Emrah Cem}

Emrah Cem received his B.S. degree in Computer Engineering and M.S. degree in Electrical and Computer Engineering from Koc University, in 2008 and 2010 , respectively. He is currently pursuing his Ph.D. degree at the University of Texas, Dallas. His research interests include statistical analysis of large networks, large scale data analysis, and distributed systems. 


\section{The Biography of Kamil Sarac}

Kamil Sarac received the M.S. and Ph.D. degrees in computer science from the University of California at Santa Barbara, in 1997 and 2002, respectively. He is currently an Associate Professor in the Department of Computer Science at the University of Texas, Dallas. His research interests include Computer networks; network and service monitoring and Internet measurements; overlay networks; network security and denial-of-service defense; broadcast in ad hoc networks; group communication and IP multicast. 


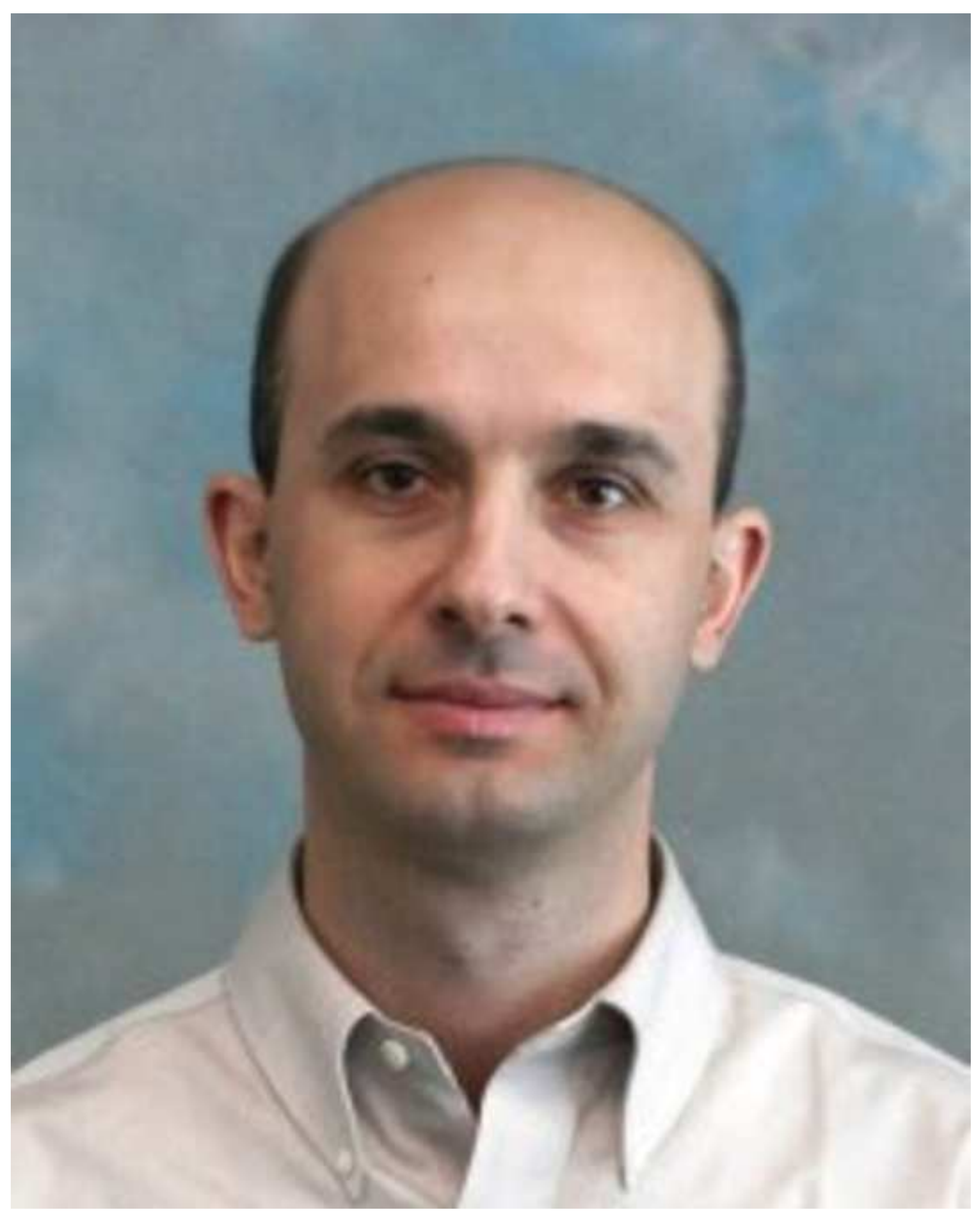

\section{Kamil Sarac}
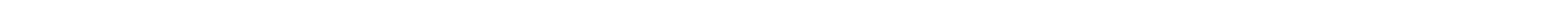


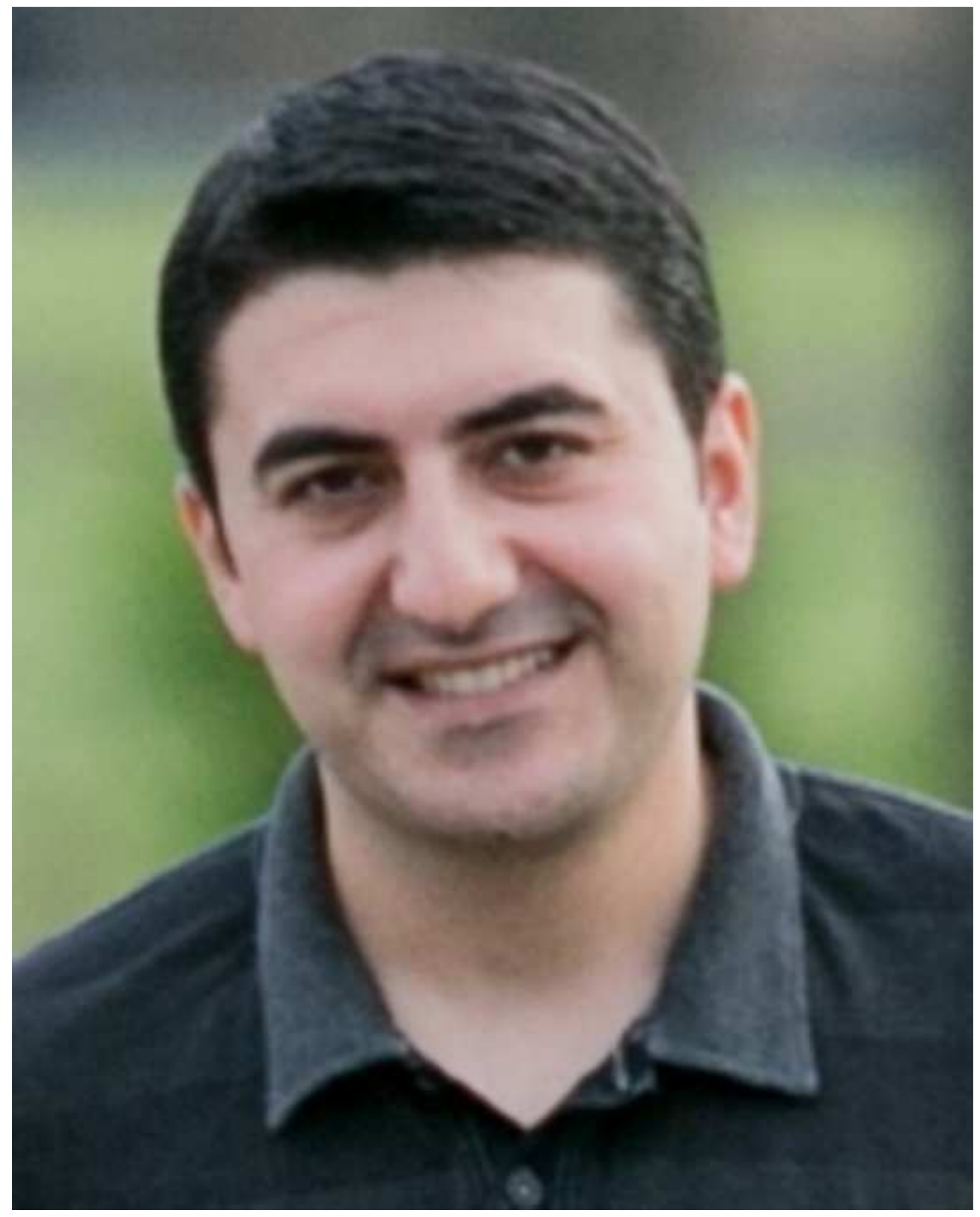

